\title{
Introduction to motives
}

\author{
Sujatha Ramdorai and Jorge Plazas \\ With an appendix by Matilde Marcolli
}

\begin{abstract}
.
This article is based on the lectures of the same tittle given by the first author during the instructional workshop of the program "number theory and physics" at ESI Vienna during March 2009. An account of the topics treated during the lectures can be found in [24] where the categorical aspects of the theory are stressed. Although naturally overlapping, these two independent articles serve as complements to each other. In the present article we focus on the construction of the category of pure motives starting from the category of smooth projective varieties. The necessary preliminary material is discussed. Early accounts of the theory were given in Manin [21] and Kleiman [19], the material presented here reflects to some extent their treatment of the main aspects of the theory. We also survey the theory of endomotives developed in [5], this provides a link between the theory of motives and tools from quantum statistical mechanics which play an important role in results connecting number theory and noncommutative geometry. An extended appendix (by Matilde Marcolli) further elaborates these ideas and reviews the role of motives in noncommutative geometry.
\end{abstract}

\section{Introduction}

Various cohomology theories play a central role in algebraic geometry, these cohomology theories share common properties and can in some cases be related by specific comparison morphisms. A cohomology theory with coefficients in a ring $R$ is given by a contra-variant functor $H$ from the category of algebraic varieties over a field $k$ to the category of graded $R$-algebras (or more generally to a $R$-linear tensor category). The functor $H$ should satisfy certain properties, in particular algebraic cycles on a variety $X$ should give rise to elements in $H(X)$ and the structure of algebraic cycles on $X$ together with their intersection product should be reflected in the structure of $H(X)$. Étale cohomology, de Rham cohomology, Betti cohomology and crystalline cohomology are examples of cohomology theories. Abstracting the formal properties shared by these cohomology theories leads to the notion of a Weil cohomology theory for which the above theories provide examples.

The idea of a universal cohomology theory for algebraic varieties led Grothendieck to the formulation of the theory of motives. Heuristically speaking, given an algebraic variety $X$ over a field $k$, the motive of $X$ should be an essential object underlying the structure shared by $H(X)$ for various cohomology theories and therefore containing the arithmetic information encoded by algebraic cycles on 
$X$. In order to develop a theory of motives, one should then construct a contravariant functor $h$ from the category of algebraic varieties over $k$ to a category $\mathcal{M}(k)$ through which any cohomology theory will factor. Thus for any Weil cohomology theory $H$, there should be a realization functor $\Upsilon_{H}$ defined on $\mathcal{M}(k)$ such that for any algebraic variety $X$ one has $H(X)=\Upsilon_{H}(h(X))$.

In these notes we will concentrate on motives of smooth projective varieties over an arbitrary base field $k$, these are called pure motives. The construction of the category of pure motives depends on the choice of an equivalence relation on algebraic cycles on varieties over $k$. We summarize here the main steps of this construction leaving the details to Chapter 2. Given such an equivalence relation $\sim$ satisfying certain properties, it is possible to enlarge the class of morphisms in the category of smooth projective varieties over $k$ in order to include $\sim$-correspondences thereby linearizing it to an additive category $\mathbf{C o r r}_{\sim}(k)$. By taking the pseudo-abelian envelope of $\operatorname{Corr}_{\sim}(k)$ one obtains the category of effective motives over $k$, denoted by $\operatorname{Mot}_{\sim}^{e f f}(k)$. The product in the category of varieties induces a tensor structure in $\operatorname{Mot}_{\sim}^{e f f}(k)$ with identity $\mathbf{1}_{k}$ corresponding to $\operatorname{Spec}(k)$. The projective line $\mathbb{P}_{k}^{1}$ decomposes in $\operatorname{Mot}_{\sim}^{e f f}(k)$ as $\mathbf{1}_{k} \oplus \mathbb{L}_{k}$ where $\mathbb{L}_{k}$ is the Lefschetz motive. The category of pure motives $\operatorname{Mot}_{\sim}(k)$ is obtained from $\operatorname{Mot}_{\sim}^{e f f}(k)$ by formally inverting $\mathbb{L}_{k}$. The functor $h$ from the category of smooth projective varieties over $k$ to $\operatorname{Mot}_{\sim}(k)$ obtained by composition of the above embeddings is called the functor of motivic cohomology. Some of the properties of the category $\operatorname{Mot}_{\sim}(k)$ and the extend to which the category depends on the choice of $\sim$ remain largely conjectural. Particular conjectures relating algebraic cycles to cohomology theories, known as the standard conjectures, were introduced by Grothendieck in the sixties partly aiming at giving the basis for the theory of motives (see [20]). The validity of these conjectures would in particular imply that the functor $h$ is itself a cohomology theory.

The contents of the paper are as follows. After recalling the necessary background in Chapter 1 we review the main steps of the construction of the category of pure motives in Chapter 2. Chapter 3 is devoted to Artin motives. A quick view of more advanced topics in Chapter 4 precedes a review of the theory of endomotives in Chapter 5. The second part of the article consists of an extended appendix (by Matilde Marcolli) surveying the role of motives in noncommutative geometry.

\section{Preliminaries}

Throughout this section we fix a base field $k$.

1.1 Cycles and correspondences. Let $X$ be an smooth projective variety over $k$. A prime algebraic cycle on $X$ is by definition a closed irreducible subvariety of $X$. Denote by $C(X)$ the free abelian group generated by prime algebraic cycles on $X$ and by $C^{r}(X)$ the subgroup of $C(X)$ generated by prime algebraic cycles 
of codimension $r$ in $X$. An element $Z \in C(X)$ is called an algebraic cycle, if $Z \in C^{r}(X)$ we say that $Z$ is an algebraic cycle of codimension $r$ on $X$. Any cycle $Z \in C^{r}(X)$ can therefore be written as a finite formal linear combination $Z=\sum n_{i} Z_{i}$ where $n_{i} \in \mathbb{Z}$ and each $Z_{i}$ is a closed irreducible subvariety of $X$ of codimension $r$.

Let $Z_{1}$ and $Z_{2}$ be two prime cycles on $X$. We say that $Z_{1}$ and $Z_{2}$ intersect properly if $\operatorname{codim}\left(Z_{1} \cap Z_{2}\right)=\operatorname{codim}\left(Z_{1}\right)+\operatorname{codim}\left(Z_{2}\right)$. In this case we can define an algebraic cycle $Z_{1} \bullet Z_{2}$ of codimension $\operatorname{codim}\left(Z_{1}\right)+\operatorname{codim}\left(Z_{2}\right)$ on $X$ as a linear combination of the irreducible components of $Z_{1} \cap Z_{2}$ with coefficients given by the intersection multiplicities (cf. [12]). More generally two algebraic cycles $Z_{1}, Z_{2} \in$ $C(X)$ intersect properly if every prime cycle in $Z_{1}$ intersects properly with every prime cycle in $Z_{2}$ in which case we obtain a well defined cycle $Z_{1} \bullet Z_{2}$ extending by linearity the above definition.

The intersection product $\bullet$ gives a partially defined multiplication from $C^{r}(X) \times$ $C^{r^{\prime}}(X)$ to $C^{r+r^{\prime}}(X)$ which is compatible with the abelian group structure on $C(X)$. In oder to obtain a graded ring starting from cycles and reflecting the geometric properties of their intersections it is necessary to impose an appropriate equivalence relation in such a way that $\bullet$ induces a well defined multiplication. There are various possible choices for such an equivalence relation leading to corresponding rings of cycles. Before analyzing these in a more systematic way it is useful to study the functoriality properties of algebraic cycles.

Let $\varphi: X \rightarrow Y$ be a morphism between two smooth projective varieties over $k$. Let $Z$ be a prime cycle on $X$. Since $\varphi$ is proper $W=\varphi(Z)$ is a closed irreducible subvariety of $Y$. If $\operatorname{dim} Z=\operatorname{dim} W$ then the function field $k(Z)$ is a finite extension of $k(W)$. Let $d=[k(Z): k(W)]$ be the degree of the extension $k(W) \hookrightarrow k(Z)$ if $\operatorname{dim} Z=\operatorname{dim} W$ and set $d=0$ otherwise. Then the map $\varphi_{*}: Z \mapsto d W$ extends by linearity to a group homomorphism

$$
\varphi_{*}: C^{r}(X) \rightarrow C^{r+(n-m)}(Y)
$$

where $m=\operatorname{dim} X$ and $n=\operatorname{dim} Y$. We call $\varphi_{*}$ the push-forward of $\varphi$.

For $\varphi$ as above let $\Gamma(\varphi) \subset X \times Y$ denote the graph subvariety of $\varphi$. If $W$ is a cycle in $Y$ such that $(X \times W) \bullet \Gamma(\varphi)$ is defined we identify this product with a cycle $Z=\varphi^{*}(W)$ on $X$ via the isomorphism $X \simeq \Gamma(\varphi)$. If $W$ is a prime cycle on $Y$ then $\varphi^{*}(W)$ is a linear combination of the irreducible components of $\varphi^{-1}(W)$. Moreover, if $\varphi$ is flat of constant relative dimension then $\varphi^{*}(W)=\varphi^{-1}(W)$. The operator $\varphi^{*}$ is linear and multiplicative whenever the appropriate cycles are defined. We call $\varphi^{*}$ the pull-back of $\varphi$.

The two maps $\varphi^{*}$ and $\varphi_{*}$ are related by the projection formula

$$
\varphi_{*}\left(\varphi^{*}(W) \bullet Z\right)=W \bullet \varphi_{*}(Z)
$$

which holds for any cycles $Z$ on $X$ and $W$ on $Y$ for which $\varphi^{*}(W) \bullet Z$ and $W \bullet \varphi_{*}(Z)$ are defined. 
Let $\sim$ be an equivalence relation defined on algebraic cycles on smooth projective varieties over $k$. The equivalence relation $\sim$ is called adequate if it satisfies the following properties (cf. $[23,19,16])$ :

- The equivalence relation $\sim$ is compatible with addition of cycles.

- If $Z_{1}$ and $Z_{2}$ are two algebraic cycles on $X$ then there exists a cycle $Z_{2}^{\prime} \in$ $C(X)$ such that $Z_{2} \sim Z_{2}^{\prime}$ and $Z_{1}$ intersects properly with $Z_{2}^{\prime}$.

- Let $X$ and $Y$ be two smooth projective varieties over $k$. Denote by $\operatorname{pr}_{2}$ the projection morphism from $X \times Y$ to $Y$. Let $Z$ be a cycle on $X$ and $W$ be a cycle on $X \times Y$ such that $W \bullet(Z \times Y)$ is defined. Then $Z \sim 0$ in $C(X)$ implies $\left(\operatorname{pr}_{2}\right)_{*}(W \bullet(Z \times Y)) \sim 0$ in $C(Y)$.

In short $\sim$ is an adequate equivalence relation if pull-back, push-forward and intersection of cycles are well defined modulo $\sim$. If $\sim$ is an adequate equivalence relation on cycles then for any smooth projective variety $X$ over $k$ the residue classes of $\sim$ form a ring under intersection product:

$$
\begin{aligned}
A_{\sim}(X) & :=C(X) / \sim \\
& =\bigoplus_{r} A_{\sim}^{r}(X), \quad \text { where } A_{\sim}^{r}(X):=C^{r}(X) / \sim
\end{aligned}
$$

Given a morphism $\varphi: X \rightarrow Y$ of smooth projective varieties the pull-back and push-forward operations on cycles induce a multiplicative operator

$$
\varphi^{*}: A_{\sim}(Y) \rightarrow A_{\sim}(X)
$$

and an additive operator

$$
\varphi_{*}: A_{\sim}^{r}(X) \rightarrow A_{\sim}^{r+(n-m)}(Y)
$$

where $m=\operatorname{dim} X$ and $n=\operatorname{dim} Y$.

Example 1.1. Let $X$ be a smooth projective variety over $k$. Two cycles $Z_{1}$ and $Z_{2}$ on $X$ are said to be rationally equivalent if there exists an algebraic cycle $W$ on $X \times \mathbb{P}^{1}$ such that $Z_{1}$ is the fiber of $W$ over 0 and $Z_{2}$ is the fiber of $W$ over 1 . We denote the resulting equivalence relation on cycles by $\sim_{\text {rat }}$. The fact that $\sim_{\text {rat }}$ is an adequate equivalence relation is a consequence of Chow's moving lemma (see $[19,12])$. For a variety $X$ the ring $A_{\mathrm{rat}}(X)$ is called the Chow ring of $X$.

Example 1.2. Let $X$ be a smooth projective variety over $k$. Two cycles $Z_{1}$ and $Z_{2}$ on $X$ are said to be algebraically equivalent if there exists an irreducible curve $T$ over $k$ and a cycle $W$ on $X \times T$ such that $Z_{1}$ is the fiber of $W$ over $t_{1}$ and $Z_{2}$ is the fiber of $W$ over $t_{2}$ for two points $t_{1}, t_{2} \in T$. We denote the resulting equivalence relation on cycles by $\sim_{\text {alg. }}$. As above $\sim_{\text {alg }}$ is an adequate equivalence relation. 
Example 1.3. Let $H$ be a Weil cohomology theory on smooth projective varieties over $k$ with coefficients in a field $F$ of characteristic 0 (see Section 1.2). Let $X$ be a smooth projective variety over $k$ with corresponding cycle class map $\mathrm{cl}_{X}$. Two cycles $Z_{1}, Z_{2} \in C^{r}(X)$ are said to be homologically equivalent with respect to $H$ if $\mathrm{cl}_{X}\left(Z_{1}\right)=\mathrm{cl}_{X}\left(Z_{2}\right)$. We denote the resulting equivalence relation on cycles by $\sim_{\text {hom. }}$. Homological equivalence is an adequate equivalence relation for any Weil cohomology theory.

Example 1.4. Let $X$ be a smooth projective variety of dimension $n$ over $k$. Two cycles $Z_{1}, Z_{2} \in C^{r}(X)$ are said to be numerically equivalent if for any $W \in$ $C^{n-r}(X)$ for which $Z_{1} \bullet W$ and $Z_{2} \bullet W$ are defined we have $Z_{1} \bullet W=Z_{2} \bullet W$. We denote the resulting equivalence relation on cycles by $\sim_{n u m}$. Numerical equivalence is an adequate equivalence relation.

Remark 1.5. (cf. $[19,16])$ Let $X$ be a smooth projective variety over $k$. Given any adequate equivalence relation $\sim$ on algebraic cycles on smooth projective varieties over $k$ there exist canonical morphisms:

$$
A_{\text {rat }}(X) \rightarrow A_{\sim}(X)
$$

and

$$
A_{\sim}(X) \rightarrow A_{\text {num }}(X)
$$

Rational equivalence is therefore the finest adequate equivalence relation for algebraic cycles on smooth projective varieties over $k$. Likewise numerical equivalence is the coarsest (non-zero) adequate equivalence relation for algebraic cycles on smooth projective varieties over $k$.

The following simple result will be used later (see $[19,21])$ :

Lemma 1.6. Let $\sim$ be an adequate equivalence relation on algebraic cycles on smooth projective varieties over $k$. Choose a rational point in $\mathbb{P}_{k}^{1}$ and denote by $e$ its class modulo $\sim$. Then

$$
A_{\sim}\left(\mathbb{P}_{k}^{1}\right)=\mathbb{Z} \oplus \mathbb{Z} e
$$

1.2 Weil cohomology theories. Denote by $\mathcal{V}(k)$ the category of smooth projective varieties over $k$. Let $F$ be a field of characteristic 0 and denote by $\mathrm{GrAlg}_{F}$ the category of graded $F$-algebras. Consider a contravariant functor

$$
\begin{aligned}
H: \mathcal{V}(k)^{o p} & \rightarrow \operatorname{GrAlg}_{F} \\
X & \mapsto H(X)=\bigoplus_{r \geq 0} H^{r}(X)
\end{aligned}
$$

For any morphism $\varphi: X \rightarrow Y$ in $\mathcal{V}(k)$ denote $H(\varphi): H(Y) \rightarrow H(X)$ by $\varphi^{*}$. Assume moreover that there exists a covariant operator from morphisms $\varphi: X \rightarrow$ 
$Y$ to linear maps $\varphi_{*}: H(X) \rightarrow H(Y)$. The fact that we use the same notations than for the induced pull-back and push-forward maps at the level of cycles should not cause any confusion. A functor $H$ as above is a Weil cohomology theory with coefficients in $F$ if it satisfies the following properties (cf. [19]):

- Given a variety $X$ in $\mathcal{V}(k)$ the space $H^{r}(X)$ is finite dimensional for any $r \geq 0$. If $r>2 \operatorname{dim} X$ then $H^{r}(X)=0$.

- Let $P=\operatorname{Spec}(k)$. Then there exists an isomorphism $a: H(P) \rightarrow F$.

- For any morphism $\varphi: X \rightarrow Y$ in $\mathcal{V}(k)$ and any $x \in H(X), y \in H(Y)$ the projection formula

$$
\varphi_{*}\left(\varphi^{*}(y) x\right)=y \varphi_{*}(x)
$$

holds.

- For any $X$ and $Y$ in $\mathcal{V}(k)$ :

$$
H(X \amalg Y) \simeq H(X) \oplus H(Y)
$$

- For any $X$ and $Y$ in $\mathcal{V}(k)$ the Kunneth formula:

$$
H(X \times Y) \simeq H(X) \otimes H(Y)
$$

holds.

- For any $X$ in $\mathcal{V}(k)$ let $\varphi_{X}: P=\operatorname{Spec}(k) \rightarrow X$ be the structure morphism and define the degree map \langle\rangle$: H(X) \rightarrow F$ as the composition $a \circ \varphi_{X}^{*}$. Then the pairing

$$
\begin{aligned}
H(X) \otimes H(X) & \rightarrow F \\
x_{1} \otimes x_{2} & \mapsto\left\langle x_{1} x_{2}\right\rangle
\end{aligned}
$$

is nondegenerate.

- For every smooth projective variety over $k$ there exists a group homomorphism $\operatorname{cl}_{X}: C(X) \rightarrow H(X)$, the cycle class map, satisfying:

(1) The map:

$$
\operatorname{cl}_{\operatorname{Spec}(k)}: C(\operatorname{Spec}(k)) \simeq \mathbb{Z} \rightarrow H(\operatorname{Spec}(k)) \simeq F
$$

is the canonical homomorphism.

(2) For any morphism $\varphi: X \rightarrow Y$ in $\mathcal{V}(k)$ its pull-back and push-forward commute with the cycle class map:

$$
\begin{aligned}
\varphi^{*} \mathrm{cl}_{Y} & =\operatorname{cl}_{X} \varphi^{*} \\
\varphi_{*} \mathrm{cl}_{X} & =\operatorname{cl}_{Y} \varphi_{*}
\end{aligned}
$$


(3) Let $X$ and $Y$ be varieties in $\mathcal{V}(k)$, let $Z \in C(X)$ and $W \in C(Y)$. Then

$$
\begin{aligned}
& \operatorname{cl}_{X \amalg Y}(Z \amalg W)=\operatorname{cl}_{X}(Z) \oplus \operatorname{cl}_{Y}(W) \\
& \operatorname{cl}_{X \times Y}(Z \times W)=\operatorname{cl}_{X}(Z) \otimes \operatorname{cl}_{Y}(W)
\end{aligned}
$$

Example 1.7. Let $k$ be a field of characteristic zero together with an embedding $k \hookrightarrow \mathbb{C}$. The Betti cohomology of a variety $X$ in $\mathcal{V}(k)$ is defined as the singular cohomology of $X(\mathbb{C})$ with coefficients in $\mathbb{Q}$. Betti cohomology is a Weil cohomology theory with coefficients in $\mathbb{Q}$.

Example 1.8. Let $k$ be a field of characteristic zero. The de Rham cohomology of a variety $X$ over $k$ can be defined in terms of the hypercohomology of its algebraic de Rham complex (see [14]). De Rham cohomology is a Weil cohomology theory with coefficients in $k$.

Example 1.9. Let $k$ be a field of characteristic $p>0$ and let $l \neq p$ be a prime number. The étale cohomology of a variety $X$ in $\mathcal{V}(k)$ is defined as the l-adic cohomology of $X \times_{\operatorname{Spec}(k)} \operatorname{Spec}(\bar{k})$. Étale cohomology is a Weil cohomology theory with coefficients in $\mathbb{Q}_{l}$.

Example 1.10. Let $k$ be a field of characteristic $p>0$. Crystalline cohomology was introduced by Grothendieck and developed by Berthelot as a substitute for ladic étale cohomology in the $l=p$ case (see [15]). Let $W(k)$ be the ring of Witt vectors with coefficients in $k$ and let $F_{\mathrm{Witt}(k)}$ be its field of fractions. Crystalline cohomology is a Weil cohomology theory with coefficients in $F_{\mathrm{Witt}(k)}$.

Remark 1.11. It is possible to define cohomology theories in a more general setting where the functor $H$ takes values on a linear tensor category $\mathcal{C}$. Properties analogous to the aforementioned ones should then hold. In particular $H$ should be a symmetric monoidal functor from $\mathcal{V}(k)$ to $\mathcal{C}$ where we view $\mathcal{V}(k)$ as a symmetric monoidal category with product $X \times Y=X \times_{\text {speck }} Y$ (this is just the Kunneth formula).

1.3 Correspondences. Let $\sim$ be a fixed adequate equivalence relation on algebraic cycles on smooth projective varieties over $k$.

Definition 1.12. Let $X$ and $Y$ be two varieties in $\mathcal{V}(k)$. An element $f \in A_{\sim}(X \times$ $Y$ ) is called a correspondence between $X$ and $Y$.

Note that this definition depends on the choice of the adequate equivalence relation $\sim$.

Given varieties $X_{1}, X_{2}$ and $X_{3}$ denote by

$$
\operatorname{pr}_{i, j}: X_{1} \times X_{2} \times X_{3} \rightarrow X_{i} \times X_{j} \quad 1 \leq i<j \leq 3
$$

the projection morphisms. Given two correspondences $f \in A_{\sim}\left(X_{1} \times X_{2}\right)$ and $g \in A_{\sim}\left(X_{2} \times X_{3}\right)$ we define their composition as the correspondence:

$$
g \circ f=\operatorname{pr}_{1,3 *}\left(p r_{1,2}^{*}(f) p r_{2,3}^{*}(g)\right) \in A_{\sim}\left(X_{1} \times X_{3}\right)
$$


It can be shown that composition of correspondences is associative for any adequate equivalence relation (cf. [21]).

For a variety $X$ in $\mathcal{V}(k)$ we denote by $\Delta_{X}$ the class of the diagonal cycle $X \hookrightarrow$ $X \times X$ in $A_{\sim}(X \times X)$. If $X$ and $Y$ are two varieties in $\mathcal{V}(k)$ and $f \in A_{\sim}(X \times Y)$, $g \in A_{\sim}(Y \times X)$ are correspondences then:

$$
f \circ \Delta_{X}=f, \quad \Delta_{X} \circ g=g
$$

Thus $\Delta_{X}$ behaves as the identity with respect to composition of correspondences.

For any morphism $\varphi: X \rightarrow Y$ denote by $\widetilde{\Gamma}_{\varphi}$ the correspondence in $A_{\sim}(X \times Y)$ given by the class of the cycle $\Gamma_{\varphi} \hookrightarrow X \times Y$ given by the graph of $\varphi$.

\section{From varieties to pure motives}

As in the previous section let $k$ be a fixed base field and denote by $\mathcal{V}(k)$ the category of smooth projective varieties over $k$. Throughout this section we fix an adequate equivalence relation $\sim$ for algebraic cycles on varieties in $\mathcal{V}(k)$. In this section we will use the formalism developed in Section 1.3 to "linearize" the category $\mathcal{V}(k)$.

2.1 Linearization. Correspondences between varieties possess all the formal properties of morphisms, we can therefore construct a new category whose objects correspond to smooth projective varieties over $k$ but whose morphisms are given by correspondences. Since cycles modulo an adequate equivalence relation form an abelian group, the category thus obtained will have the advantage of being an additive category.

Given two varieties $X$ and $Y$ in $\mathcal{V}(k)$ we set

$$
\operatorname{Corr}_{\sim}(X, Y)=\bigoplus_{i} A_{\sim}^{\operatorname{dim} X_{i}}(X \times Y)
$$

where $X_{i}$ are the irreducible components of $X$.

Definition 2.1. Let $F$ be a field of characteristic 0. We define $\operatorname{Corr}_{\sim}(k, F)$, the category of correspondences over $k$ with coefficients in $F$, as the category whose objects are smooth projective varieties over $k$ :

$$
\operatorname{Obj}\left(\operatorname{Corr}_{\sim}(k, F)\right)=\operatorname{Obj}(\mathcal{V}(k))
$$

and whose morphisms are given by:

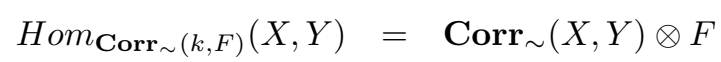

Composition of morphisms is given by composition of correspondences. The identity morphism in $\operatorname{Hom}_{\mathrm{Corr}(k)}(X, X)$ is given by the correspondence $\Delta_{X}$. 
It follows from the definitions that given any adequate equivalence relation $\sim$ for algebraic cycles on varieties in $\mathcal{V}(k)$ the category $\operatorname{Corr}_{\sim}(k, F)$ is an $F$-linear category. We denote $\operatorname{Corr}_{\sim}(k, \mathbb{Q})$ by $\operatorname{Corr}_{\sim}(k)$ and refer to it simply as the category of correspondences over $k$.

The category $\mathcal{V}(k)$ can be faithfully embedded into the category $\operatorname{Corr}_{\sim}(k, F)$ via the contravariant functor

$$
h: \mathcal{V}(k) \rightarrow \operatorname{Corr}_{\sim}(k, F)
$$

which acts as the identity on objects and sends a morphism $\varphi: Y \rightarrow X$ in $\mathcal{V}(k)$ to the correspondence $\widetilde{\Gamma}_{\varphi}$ in $\operatorname{Hom}_{\mathbf{C o r r}_{\sim}(k, F)}(X, Y)$ (see 1.3). We denote therefore by $h(X)$ a smooth projective variety $X$ when considered as an object in $\operatorname{Corr}_{\sim}(k, F)$.

The product of varieties in $\mathcal{V}(k)$ induces a tensor structure in the category $\operatorname{Corr}_{\sim}(k, F)$ via:

$$
h(X) \otimes h(Y)=h(X \times Y)
$$

turning $\mathbf{C o r r}_{\sim}(k, F)$ into a $F$-linear tensor category with identity object given by

$$
\mathbf{1}_{k}:=h(\text { Speck })
$$

2.2 Pseudo-abelianization. The category $\operatorname{Corr}_{\sim}(k, F)$ obtained in Section 2.1, although being $F$-linear, is still far from abelian. In particular not every idempotent morphism in $\operatorname{Corr}_{\sim}(k, F)$ corresponds to a direct sum decomposition of the underlying object. In this section, we will formally add the kernels of idempotent morphisms in $\operatorname{Corr}_{\sim}(k, F)$ in order to obtain a pseudo-abelian category. As this formal procedure can be carried out for any additive category, we start this section by describing it in this generality.

Definition 2.2. An additive category $\mathcal{A}$ is called pseudo-abelian if for any object $A$ in $\mathcal{A}$ and any idempotent endomorphism $p=p^{2} \in \operatorname{Hom}_{\mathcal{A}}(A, A)$ there exist a kernel ker $p$ and the canonical morphism:

$$
\operatorname{ker} p \oplus \operatorname{ker}\left(i d_{A}-p\right) \rightarrow A
$$

is an isomorphism.

Given any additive category $\mathcal{D}$, it is possible to construct a pseudo-abelian category $\widetilde{\mathcal{D}}$ into which $\mathcal{D}$ embeds fully faithfully via a functor

$$
\Psi_{\mathcal{D}}: \mathcal{D} \rightarrow \widetilde{\mathcal{D}}
$$

which is universal in the sense that given any additive functor $F: \mathcal{D} \rightarrow \mathcal{A}$ where $\mathcal{A}$ is a pseudo-abelian category there exists an additive functor $\tilde{F}: \widetilde{\mathcal{D}} \rightarrow \mathcal{A}$ such that the functors $G$ and $\tilde{F} \Psi_{\mathcal{D}}$ are equivalent. 
The category $\widetilde{\mathcal{D}}$ is obtained by formally adding kernels of idempotent endomorphisms in $\mathcal{D}$. Objects in the category $\mathcal{\mathcal { D }}$ are given by pairs $(D, p)$ where $D$ is an object in $\mathcal{D}$ and $p=p^{2} \in \operatorname{Hom}_{\mathcal{D}}(D, D)$ is an idempotent endomorphism:

$$
\operatorname{Obj}(\widetilde{\mathcal{D}})=\left\{(D, p) \mid D \in \operatorname{Obj}(\mathcal{D}), p=p^{2} \in \operatorname{Hom}_{\mathcal{D}}(D, D)\right\}
$$

If $(D, p)$ and $\left(D^{\prime}, p^{\prime}\right)$ are objects in $\widetilde{\mathcal{D}}$ we define $\operatorname{Hom}_{\widetilde{\mathcal{D}}}\left((D, p),\left(D^{\prime}, p^{\prime}\right)\right)$ to be the quotient group

$$
\frac{\left\{f \in \operatorname{Hom}_{\mathcal{D}}\left(D, D^{\prime}\right) \text { such that } f p=p^{\prime} f\right\}}{\left\{f \in \operatorname{Hom}_{\mathcal{D}}\left(D, D^{\prime}\right) \text { such that } f p=p^{\prime} f=0\right\}}
$$

Composition of morphisms is induced from composition of morphisms in $\mathcal{D}$. The category thus obtained is a pseudo-abelian category and the functor given on objects by

$$
\Psi_{\mathcal{D}}: D \mapsto\left(D, \mathrm{id}_{D}\right)
$$

and sending a morphism $f \in \operatorname{Hom}_{\mathcal{D}}\left(D, D^{\prime}\right)$ to its class in $\operatorname{Hom}_{\mathcal{D}}\left(D, D^{\prime}\right) /(0)$ is fully faithful and satisfies the above universal property.

We call $\widetilde{\mathcal{D}}$ the pseudo-abelian envelope of $\mathcal{D}$ (also sometimes referred to as the idempotent completion of $\mathcal{D}$, or the Karoubi envelope $\mathcal{D}$ ). If the category $\mathcal{D}$ is an $F$-linear category for a field $F$ then $\widetilde{\mathcal{D}}$ is an $F$-linear pseudo-abelian category. If the category $\mathcal{D}$ has an internal tensor product $\otimes$ then the product

$$
(D, p) \otimes\left(D^{\prime}, p^{\prime}\right)=\left(D \otimes D^{\prime}, p \otimes p^{\prime}\right)
$$

is an internal tensor product on $\widetilde{\mathcal{D}}$.

Definition 2.3. Let $F$ be a field of characteristic 0 . The category of effective motives over $k$ with coefficients in $F$, denoted by $\operatorname{Mot}_{\sim}^{e f f}(k, F)$, is the pseudoabelian envelope of the category $\operatorname{Corr}_{\sim}(k, F)$.

As above we denote $\operatorname{Mot}_{\sim}^{e f f}(k, \mathbb{Q})$ by $\operatorname{Mot}_{\sim}^{e f f}(k)$ and refer to its objects simply as effective motives over $k$. The category $\operatorname{Mot}_{\sim}^{e f f}(k, F)$ is by construction a pseudo-abelian $F$-linear tensor category.

We can extend the functor $h$ from $\mathcal{V}(k)$ to $\operatorname{Corr}_{\sim}(k, F)$ to a functor from $\mathcal{V}(k)$ to $\operatorname{Mot}_{\sim}^{e f f}(k, F)$ by composing it with the canonical embedding $\Psi_{\mathbf{C o r r}_{\sim}(k, F)}$, we denote the functor thus obtained also by $h$.

Spelling out the definition of the pseudo-abelian envelope in this particular case, we see that effective motives over $k$ can be represented as pairs

$$
(h(X), p)
$$

where $X$ is a smooth projective variety over $k$ and $p \in \operatorname{Corr}(X, X) \otimes F$ is an idempotent correspondence. Since for any such idempotent we have

$$
\operatorname{ker} p \oplus \operatorname{ker}\left(\Delta_{X}-p\right)=h(X)
$$


in $\operatorname{Mot}_{\sim}^{e f f}(k, F)$, we see that effective motives over $k$ are essentially given by direct factors of smooth projective varieties over $k$.

Consider the case of $\mathbb{P}_{k}^{1}$. Let $e$ be as in lemma Lemma 1.6, then the correspondence $(1 \times e) \in \operatorname{Corr}\left(\mathbb{P}_{k}^{1}, \mathbb{P}_{k}^{1}\right)$ is idempotent. We define the Lefschetz motive over $k$ to be the effective motive given by:

$$
\mathbb{L}_{k}=\left(h\left(\mathbb{P}_{k}^{1}\right),(1 \times e)\right) ;
$$

in particular we get a decomposition of $\mathbb{P}_{k}^{1}$ in $\operatorname{Mot}_{\sim}^{e f f}(k, F)$ of the form:

$$
h\left(\mathbb{P}_{k}^{1}\right)=\mathbf{1}_{k} \oplus \mathbb{L}
$$

where as above we take $\mathbf{1}_{k}=h(\operatorname{Spec}(k))$. More generally, we obtain a decomposition of $r$-dimensional projective space over $k$ as

$$
h\left(\mathbb{P}^{r}\right)=\mathbf{1} \oplus \mathbb{L}_{k} \oplus \cdots \oplus \mathbb{L}_{k}^{r}
$$

where

$$
\mathbb{L}_{k}^{i}=\mathbb{L}_{k} \otimes \cdots \otimes \mathbb{L}_{k} \quad i \text { times. }
$$

It can also be shown that an irreducible curve $X$ in $\mathcal{V}(k)$ admits a decomposition in $\operatorname{Mot}_{\sim}^{e f f}(k, F)$ of the form:

$$
h(X)=\mathbf{1} \oplus h^{1}(X) \oplus \mathbb{L}_{k} .
$$

2.3 Inversion. Tensoring with the Lefschetz motive induces a functor

$$
\begin{aligned}
M & \longmapsto M \otimes \mathbb{L}_{k} \\
f & \longmapsto f \otimes i d_{\mathbb{L}_{k}}
\end{aligned}
$$

from the category $\operatorname{Mot}_{\sim}^{e f f}(k, F)$ to itself. This functor is fully faithful. In particular, given two effective motives $M$ and $M^{\prime}$ and integers $n, m, N$ with $N \geq n, m$ the $F$-vector space

$$
\operatorname{Hom}_{\left.\mathbf{M o t}_{\sim}^{e f f}(k, F)\right)}\left(M \otimes \mathbb{L}_{k}^{N-m}, M^{\prime} \otimes \mathbb{L}_{k}^{N-n}\right)
$$

is independent of the choice of $N$. This can be used to obtain the category of pure motives from the category of effective motives by formally inverting the element $\mathbb{L}_{k}$. More precisely, define the category of pure motives $\operatorname{Mot}_{\sim}(k, F)$ as the category whose objects are given by pairs $(M, n)$ where $M$ is an effective motive and $m$ is an integer

$$
\operatorname{Obj}\left(\operatorname{Mot}_{\sim}(k, F)\right)=\left\{(M, m) \mid M \in \operatorname{Obj}\left(\operatorname{Mot}_{\sim}^{e f f}(k, F)\right), m \in \mathbb{Z}\right\}
$$

and whose morphisms are given by:

$\operatorname{Hom}_{\mathbf{M o t}_{\sim}(k, F)}\left((M, m),\left(M^{\prime}, n\right)\right)=\operatorname{Hom}_{\left.\mathbf{M o t}_{\sim}^{e f f}(k, F)\right)}\left(M \otimes \mathbb{L}_{k}^{N-m}, M^{\prime} \otimes \mathbb{L}_{k}^{N-n}\right)$ 
where $N \geq n, m$. As above we let $\operatorname{Mot}_{\sim}(k, \mathbb{Q})=\operatorname{Mot}_{\sim}(k)$.

The category $\operatorname{Mot}_{\sim}(k, F)$ has a tensor product given by

$$
(M, m) \otimes\left(M^{\prime}, n\right)=\left(M \otimes M^{\prime}, m+n\right) .
$$

We can embed the category of effective motives $\left.\operatorname{Mot}_{\sim}^{e f f}(k, F)\right)$ as a subcategory of $\operatorname{Mot}_{\sim}(k, F)$ via the functor:

$$
M \longmapsto(M, 0) .
$$

As before we denote by $h$ the functor from $\mathcal{V}(k)$ to $\operatorname{Mot}_{\sim}(k, F)$ induced by the above embedding.

Denote by $\mathbb{T}_{k}$ the object $\left(\mathbf{1}_{k},-1\right)$ in $\boldsymbol{M o t}_{\sim}(k, F)$ and write $\mathbb{T}_{k}^{n}$ for $\left(\mathbf{1}_{k},-n\right)$, $n \in \mathbb{Z}$. Then $\mathbb{T}_{k}^{0}=\mathbf{1}_{k}$ and there is a canonical isomorphism:

$$
\mathbb{T}_{k}^{-1}=\mathbb{L}_{k} .
$$

The element $\mathbb{T}_{k}$ is called the Tate motive. $\mathbb{T}_{k}$ plays a role analogous to the Tate module in $l$-adic cohomology. We define

$$
M(n)=M \otimes \mathbb{T}_{k}^{n} \quad \text { ("Tate twisting"). }
$$

Any pure motive can be written as $M(n)$ for an effective motive $M$ and an integer $n$. It can be shown (see [11]) that for any smooth projective variety $X$ there are canonical isomorphisms:

$$
A_{\sim}^{r}(X) \otimes F \simeq \operatorname{Hom}_{\mathbf{M o t}_{\sim}(k, F)}\left(\mathbf{1}_{k}, h(X)(r)\right) .
$$

As mentioned in the Introduction some of the properties of the category $\operatorname{Mot}_{\sim}(k)$ and the extent to which it depends on the choice of $\sim$ remain largely conjectural. We end this section with an important result due to Jannsen in the case $\sim$ is numerical equivalence on cycles (Example 1.4).

Theorem 2.4 (Jannsen [17]). The category $\operatorname{Mot}_{\text {num }}(k, F)$ is a semi-simple Flinear, rigid tensorial category.

\section{Artin motives}

Artin motives are motives of zero dimensional varieties, already at this level various facets of the theory make their appearance and some of the richness of the underlying structures manifests itself. In a sense which we will make more precise, the theory of Artin motives can be considered as a linearization of Galois theory.

Let $\mathcal{V}^{0}(k)$ be the subcategory of $\mathcal{V}(k)$ consisting of varieties of dimension 0 over $k$. Objects in $\mathcal{V}^{0}(k)$ are given by spectra of finite $k$-algebras;

$$
\operatorname{Obj}\left(\mathcal{V}^{0}(k)\right)=\left\{X \in \operatorname{Obj}(\mathcal{V}(k)) \mid X=\operatorname{Spec}(A), \operatorname{dim}_{k} A<\infty\right\}
$$


Fix a separable closure $k^{s e p}$ of $k$. Then for any $X=\operatorname{Spec}(A) \in \operatorname{Obj}\left(\mathcal{V}^{0}(k)\right)$ the absolute Galois group $G_{k}=\operatorname{Gal}\left(k^{\text {sep }} / k\right)$ acts continuously on the set of algebraic points of $X$ :

$$
X\left(k^{s e p}\right)=H_{o m-\operatorname{alg}}\left(A, k^{s e p}\right) .
$$

The action of $G_{k}$ commutes with morphisms in $\mathcal{V}^{0}(k)$ since these are given by rational maps. Taking algebraic points induces then a functor

$$
X \longmapsto X\left(k^{s e p}\right)
$$

between $\mathcal{V}^{0}(k)$ and the category $\mathcal{F}^{0}\left(G_{k}\right)$ consisting of finite sets endowed with a continuous $G_{k}$-action. The fact that this functor is an equivalence of categories is essentially a restatement of the main theorem of Galois theory. The inverse functor maps a finite $G_{k}$-set $I \in \operatorname{Obj}\left(\mathcal{F}^{0}\left(G_{k}\right)\right)$ to the spectrum of the ring of $G_{k}$-invariant functions from $I$ to $k^{s e p}$. This equivalence of categories is usually referred as the Grothendieck-Galois correspondence.

The category of Artin motives $\operatorname{Mot}^{0}(k, F)$, is by definition the subcategory of $\operatorname{Mot}_{\sim}(k, F)$ spanned by objects of the form $h(X)$ for $X \in \operatorname{Obj}\left(\mathcal{V}^{0}(k)\right)$. It is important to note that since any adequate equivalence relation on algebraic cycles on varieties in $\mathcal{V}(k)$ becomes trivial when restricted to $\mathcal{V}^{0}(k)$, this definition does not depend on the choice of $\sim$. Also, since the Lefschetz motive corresponds to the decomposition of the one dimensional variety $\mathbb{P}_{k}^{1}$ there is no need to take into account the twisting by $\mathbb{T}_{k}$. The category $\operatorname{Mot}^{0}(k, F)$ is therefore the pseudoabelian envelope of the category of correspondences of zero dimensional varieties $\operatorname{Corr}^{0}(k, F)$, whose objects are given by varieties in $\mathcal{V}^{0}(k)$ and whose morphisms are given by:

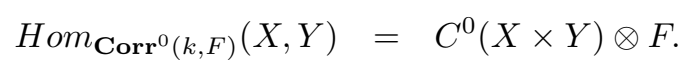

A correspondence between two varieties $X$ and $Y$ in $\mathcal{V}^{0}(k)$ (with coefficients in $F$ ) is thus given by a formal linear combination of connected components of $X \times Y$ with coefficients in $F$. By taking characteristic functions we may identify such a correspondence with a $G_{k}$-invariant function from $X\left(k^{s e p}\right) \times Y\left(k^{s e p}\right)$ to $F$. Composition of correspondences becomes matrix multiplication and passing to the pseudo-abelian envelope we get an equivalence of categories:

$$
\operatorname{Mot}^{0}(k, F) \simeq \operatorname{Rep}\left(G_{k}, F\right)
$$

where $\operatorname{Rep}\left(G_{k}, F\right)$ is the category of finite dimensional $F$-representations of the group $G_{k}$. The functor of motivic cohomology restricted to dimension zero is then given by

$$
h: X \mapsto F^{X\left(k^{s e p}\right)}
$$

where $X \in \operatorname{Obj}\left(\mathcal{V}^{0}(k)\right)$ and the $F$-vector space $F^{X\left(k^{s e p}\right)}$ is endowed with the natural $G_{k}$-action. The category $\operatorname{Mot}^{0}(k, F)$ has a rich structure coming from the 
fact that it can be identified with the category of representations of a group, the corresponding properties encoded thereby correspond to the fact that $\operatorname{Mot}^{0}(k, F)$ is a Tannakian category.

When reference to an ambient category is relevant it is customary to view $\operatorname{Mot}^{0}(k, F)$ as a subcategory of $\operatorname{Mot}_{\text {num }}(k, F)$.

\section{Vistas}

As mentioned in the introduction the formal properties in the construction of $\operatorname{Mot}_{\sim}(k, F)$ imply the existence of realization functors

$$
\Upsilon_{H}: \operatorname{Mot}_{\sim}(k, F) \rightarrow \operatorname{GrAlg}_{F}
$$

for various Weil cohomology theories $H: \mathcal{V}(k)^{o p} \rightarrow \operatorname{GrAlg}_{F}$ defined on $\mathcal{V}(k)$. These functors enrich the structure of $\operatorname{Mot}_{\sim}(k, F)$ and lead to further important constructions.

Realization functors play an important role in the definition of $L$-functions associated to motives the special values of which are of prime importance in number theory (cf. [10]).

Realization functors also play a role in the definition of motivic Galois groups. In order to be able to modify the category $\operatorname{Mot}_{\sim}(k, F)$ to obtain a category equivalent to the category of representations of a group,it is necessary to have a fiber functor with values on $F$-vector spaces playing the role of forgetful functor. As mentioned in the previous section in the case of Artin motives the absolute Galois group of the base field is recovered from this formalism. However more general cases involve in a deep way the validity of the standard conjectures (see [24] for a review). Once a Tannakian category of motives has been constructed the corresponding motivic Galois group provides a rich higher dimensional analogue of Galois theory.

From here on, the theory develops rapidly and branches in numerous directions, leading to a very rich landscape of results connecting and interrelating various areas of mathematics. The largely conjectural theory of mixed motives, that is motives of varieties which are not necessary smooth or projective, seems to underlay phenomena relevant to different fields. Areas like Hodge theory, $K$-theory and automorphic forms are enriched by the presence of structures of motivic origin leading in many cases to deep conjectures. For an account of various aspects of the theory the reader may consult the two volumes [18].

\section{Endomotives}

Various interactions between noncommutative geometry and number theory have taken place recently. The tools and methods developed by noncommutative geometry are well suited for the study of structures relevant in number theory, and in many cases shed new light into old outstanding problems (cf. $[22,6]$ ). Some 
aspects of the theory so developed make contact with the theory of motives in a natural way. In this section we describe the construction of a class of noncommutative spaces closely related to Artin motives and its relevance in the study of class field theory. The main reference for the material treated in this section is [5] which we follow closely (see also [6] [9]).

5.1 Adèles and Idèles, basics in class field theory. Global class field theory describes the abelian extensions of a global field $k$ in terms of analyitic-arithmetic data coming from the field itself. We start this section by briefly recalling some basic notions from global class field theory (cf. [1]). A global field $k$ is by definition a field of one of the following two kinds:

- a number field, i.e. a finite extension of $\mathbb{Q}$, the field of rational numbers.

- a function field, i.e. a finite extension of $\mathbb{F}_{q}(t)$, the field of rational functions in one variable over a finite field.

An important part of the structure of a global field $k$ is encoded by ideals in its ring of integers $\mathcal{O}_{k}$ which is given by the integral closure in $k$ of $\mathbb{Z}$ in the number field case or that of $\mathbb{F}_{q}[t]$ in the function field case.

A valuation on a global field $k$ is by definition a nonnegative multiplicative function || from $k$ to $\mathbb{R}$, with $|0|=0$ and non-vanishing on $k^{*}=k \backslash\{0\}$, satisfying the triangle inequality:

$$
|x+y| \leq|x|+|y| \quad \text { for all } x, y \in k .
$$

The valuation || is called non-archimedean if it satisfies

$$
|x+y| \leq \max \{|x|,|y|\} \quad \text { for all } x, y \in k,
$$

otherwise we say that the valuation || is archimedean. Two valuations on a global field $k$ are said to be equivalent if the corresponding metrics induce the same topology on $k$. A place $\nu$ on a global field $k$ is by definition an equivalence class of valuation on $k$. A place $\nu$ on $k$ is said to be archimedean (resp. non-archimedean) if it consist of archimedean (resp. non-archimedean) valuations. Given a place $\nu$ on a global field $k$ we denote by $k_{\nu}$ the completion of $k$ with respect to the metric induced by any of the valuations in $\nu$. The space $k_{\nu}$ is a locally compact field. If $\nu$ is a non-archimedean place, the set

$$
\mathcal{O}_{k, \nu}=\left\{\left.\eta \in k_{\nu}|| \eta\right|_{\nu} \leq 1\right\}
$$

is a subring of $k_{\nu}$ where ||$_{\nu}$ is the norm in $k_{\nu}$ induced by $\nu$. We call $\mathcal{O}_{k, \nu}$ the ring of integers of $k_{\nu}$. For a non-archimedean place $\nu$ on $k$ the ring $\mathcal{O}_{k, \nu}$ is an open compact subring of $k_{\nu}$.

If $k$ is a number field, then non-archimedean places on $k$ are in one to one correspondence with prime ideals in $\mathcal{O}_{k}$ while the archimedean places on $k$ correspond to the finitely many different embeddings of $k$ in $\mathbb{C}$. This is essentially a 
consequence of Ostrowski's theorem by which any valuation on $\mathbb{Q}$ is equivalent to the $p$-adic absolute value ||$_{p}$ for some prime number $p$ or to the ordinary absolute value induced by the embedding of $\mathbb{Q}$ in $\mathbb{R}$.

Given a collection $\left\{\Sigma_{\lambda}\right\}_{\lambda \in \Lambda}$ of locally compact topological spaces and compact subspaces $\Omega_{\lambda} \subset \Sigma_{\lambda}$ for all but finitely many $\lambda \in \Lambda$, there exists a unique topology on the set

$$
\prod^{(\text {res })} \Sigma_{\lambda}=\left\{\left(\eta_{\lambda}\right) \in \prod \Sigma_{\lambda} \mid \eta_{\lambda} \in \Omega_{\lambda} \text { for all but finitely many } \lambda \in \Lambda\right\}
$$

for which $\prod \Omega_{\lambda}$ is a compact subspace. We call the set $\prod^{(r e s)} \Sigma_{\lambda}$ together with this topology the restricted topological product of $\left\{\Sigma_{\lambda}\right\}_{\lambda \in \Lambda}$ with respect to the subspaces $\Omega_{\lambda}$.

Definition 5.1. Let $k$ be a global field and let $\mathcal{P}$ be the collection of places on $k$. The ring of adèles of $k$, denoted by $\mathbb{A}_{k}$, is the topological ring given by the restricted topological product of $\left\{k_{\nu}\right\}_{\nu \in \mathcal{P}}$ with respect to the subspaces $\mathcal{O}_{k, \nu}$. The group of idèles of $k$, denoted by $\mathbb{I}_{k}$, is the topological group given by the restricted topological product of $\left\{k_{\nu}^{*}\right\}_{\nu \in \mathcal{P}}$ with respect to the subspaces $\mathcal{O}_{k, \nu}^{*}$.

Any global field $k$ can be embedded as a discrete co-compact subfield of the locally compact ring $\mathbb{A}_{k}$. Likewise $k^{*}$ embeds diagonally in $\mathbb{I}_{k}$. The quotient topological group

$$
\mathcal{C}_{k}=\mathbb{I}_{k} / k^{*}
$$

is called the idèle class group of $k$.

One of the central results in class field theory associates to any abelian extension of a global field $k$ a subgroup of the idèle class group. The main theorem of global class field theory can be stated as follows:

Theorem 5.2. Let $k$ be a number field and let $k^{a b}$ be its maximal abelian extension. Denote by $\mathcal{D}_{k}$ the connected component of the identity in $\mathcal{C}_{k}$. Then there is a canonical isomorphism of topological groups:

$$
\Psi: \mathcal{C}_{k} / \mathcal{D}_{k} \rightarrow \operatorname{Gal}\left(k^{a b} / k\right)
$$

In the case of a function field $k$ an analogous result identifies in a canonical way the group $\operatorname{Gal}\left(k^{a b} / k\right)$ with the profinite completion of $\mathcal{C}_{k}$. The isomorphism $\Psi$ is usually referred as the global Artin map or reciprocity map.

5.2 Noncommutative spaces. One of the departure points of noncommutative geometry is the duality between various classes of spaces and algebras of functions canonically associated to these spaces. One classical instance of this duality is furnished by Gelfand's theorem which provides a one to one correspondence between compact Hausdorff topological spaces and unital $C^{*}$-algebras via the functor that 
sends a space $X$ to its algebra of complex valued continuous functions. Other examples and of this type of duality and refinements thereof abound in the mathematical landscape. In these situations it might be possible to define structures relevant to the study of a space in terms of the corresponding algebras of functions and, in cases in which these definitions do not depend on the commutativity of the underlying algebra, extend them in order to study noncommutative algebras. Geometric information which is difficult to encode with traditional tools might be understood by enlarging the class of spaces in consideration in such a way as to allow the presence of "noncommutative coordinates". In this way we view a noncommutative algebra as defining by duality a noncommutative space for which this algebra plays the role of algebra of coordinates. One may then for example view a noncommutative unital $C^{*}$-algebra as defining by duality a "noncommutative compact Hausdorff topological space". This process is far from being a mere translation of concepts to another framework even when such a translation might prove to be delicate. Many new phenomena arise in this context and in some situations the classical picture is also enriched (cf. [4]).

5.3 Quantum statistical mechanics. A particular area in which these ideas occur naturally is quantum statistical mechanics. A quantum statistical mechanical system is determined by a $C^{*}$-algebra $\mathcal{A}$ (the algebra of observables of the system) and a one-parameter group of automorphisms $\sigma_{t} \in \operatorname{Aut}(\mathcal{A}), t \in \mathbb{R}$ (the time evolution of the system). A state on the $C^{*}$-algebra $\mathcal{A}$ is by definition a normone linear functional $\varphi: A \rightarrow \mathbb{C}$ satisfying the positivity condition $\varphi\left(a^{*} a\right) \geq 0$ for all $a \in \mathcal{A}$. In a quantum statistical mechanical system $\left(\mathcal{A}, \sigma_{t}\right)$ the time evolution $\sigma_{t}$ singles out a class of states of the algebra $\mathcal{A}$, the equilibrium states of the system, these are families of states parametrized by a positive real number $\beta$ corresponding to a thermodynamical parameter (the inverse temperature of the system). The appropriate definition of equilibrium states in the context of quantum statistical mechanics was given by Haag, Hugenholtz and Winnink in [13]. This condition, known after Kubo, Martin and Schwinger as the $K M S$ condition, is given as follows:

Definition 5.3. Let $\left(\mathcal{A}, \sigma_{t}\right)$ be a quantum statistical mechanical system. A state $\varphi$ on $\mathcal{A}$ satisfies the $K M S$ condition at inverse temperature $0<\beta<\infty$ if for every $a, b \in \mathcal{A}$ there exists a bounded holomorphic function $F_{a, b}$ on the strip $\{z \in \mathbb{C} \mid 0<\Im(z)<\beta\}$, continuous on the closed strip, such that

$$
F_{a, b}(t)=\varphi\left(a \sigma_{t}(b)\right), \quad F_{a, b}(t+i \beta)=\varphi\left(\sigma_{t}(b) a\right), \quad \forall t \in \mathbb{R}
$$

We call such state a $K M S_{\beta}$ state. A $K M S_{\infty}$ state is by definition a weak limit of $K M S_{\beta}$ states as $\beta \rightarrow \infty$.

For each $0<\beta \leq \infty$ the set of $K M S_{\beta}$ states associated to the time evolution $\sigma_{t}$ is a compact convex space (cf. [3], Section 5.3). We denote by $\mathcal{E}_{\beta}$ the space of extremal points of the space of $K M S_{\beta}$ states. A group $G \subset A u t(\mathcal{A})$ such that 
$\sigma_{t} g=g \sigma_{t}$ for all $g \in G$ and all $t \in \mathbb{R}$ is called a symmetry group of the system $\left(\mathcal{A}, \sigma_{t}\right)$. If $G$ is a symmetry group of the system $\left(\mathcal{A}, \sigma_{t}\right)$ then $G$ acts on the space of $K M S_{\beta}$ states for any $\beta$ and hence on $\mathcal{E}_{\beta}$. Inner automorphisms coming from unitaries invariant under the time evolution act trivially on equilibrium states.

Starting with the seminal work of Bost and Connes, [2], various quantum statistical mechanical systems associated to arithmetic data have been studied. Potential applications to the explicit class field theory problem make the understanding of such systems particularly valuable (see $[22,6]$ and references therein). We recall below the definition and properties of the quantum statistical mechanical system introduced in [2] in the form most adequate for our purposes, which in particular serves as a motivating example for the introduction of endomotives.

5.4 The Bost-Connes system. For a positive integer $n$ consider the cyclic group of order $n$ as a zero dimensional variety over $\mathbb{Q}$ given by

$$
X_{n}=\operatorname{Spec}\left(A_{n}\right) ; \quad A_{n}=\mathbb{Q}[\mathbb{Z} / n \mathbb{Z}]
$$

We order $\mathbb{N}^{\times}$by divisibility. For $n \mid m$ the canonical morphism $X_{m} \rightarrow X_{n}$ is a morphism in $\mathcal{V}^{0}(\mathbb{Q})$ and we can view $\left\{X_{n}\right\}_{n \in \mathbb{N}^{\times}}$as a projective system of zero dimensional algebraic varieties over $\mathbb{Q}$. The pro-finite limit

$$
X=\lim _{n} X_{n}
$$

corresponds to the direct limit of finite dimensional algebras:

$$
\begin{aligned}
A & =\underset{\rightarrow}{\lim _{n} A_{n}} \\
& =\underset{\rightarrow}{\lim } \mathbb{Q}[\mathbb{Z} / n \mathbb{Z}] \\
& \simeq \mathbb{Q}[\mathbb{Q} / \mathbb{Z}]
\end{aligned}
$$

Denote by $\{e(r) \mid r \in \mathbb{Q} / \mathbb{Z}\}$ the canonical basis of $A$. The multiplicative abelian semigroup $\mathbb{N}^{\times}$acts as a semigroup of endomorphisms of the algebra $A$ by:

$$
\begin{aligned}
\rho_{n}: A & \rightarrow A \\
e(r) & \mapsto \frac{1}{n} \sum_{l \in \mathbb{Q} / \mathbb{Z} ; l n=r} e(l)
\end{aligned}
$$

The algebra of continous functions on the pro-finite space $X(\overline{\mathbb{Q}})$ coincides with $C^{*}(\mathbb{Q} / \mathbb{Z})$, the group $C^{*}$-algebra of $\mathbb{Q} / \mathbb{Z}$ (i.e. the completion of $\mathbb{C}[\mathbb{Q} / \mathbb{Z}]$ in its regular representation). The semigroup action of $\mathbb{N}^{\times}$on $A$ extends to an action of semigroup action of $\mathbb{N}^{\times}$on $C^{*}(\mathbb{Q} / \mathbb{Z})$ and the semigroup crossed product algebra $\mathcal{A}_{\mathbb{Q}}=A \rtimes \mathbb{N}^{\times}$is a rational sub-algebra of the the semigroup crossed product $C^{*}$-algebra

$$
\mathcal{A}=C^{*}(\mathbb{Q} / \mathbb{Z}) \rtimes \mathbb{N}^{\times}
$$


The $C^{*}$-algebra $\mathcal{A}$ coincides with the $C^{*}$-algebra generated by elements $e(r), r \in$ $\mathbb{Q} / \mathbb{Z}$ (corresponding the generators of the group algebra of $\mathbb{Q} / \mathbb{Z}$ ) and $\mu_{n}, n \in \mathbb{N}^{\times}$ (corresponding the generators of the semigroup action) and satisfying the relations:

$$
\begin{array}{rc}
e(0)=1, e(r) e(s)=e(r+s), e(r)^{*}=e(-r), & \text { for all } r, s \mathbb{Q} / \mathbb{Z} \\
\mu_{n} \mu_{k}=\mu_{n k}, \mu_{n}^{*} \mu_{n}=1 & \text { for all } n, k \in \mathbb{N}^{\times} \\
\mu_{n} e(r) \mu_{n}^{*}=\rho_{n}(e(r)) & \text { for all } n \in \mathbb{N}^{\times}, r \in \mathbb{Q} / \mathbb{Z}
\end{array}
$$

It is possible to define a time evolution $\sigma_{t}$ on the $C^{*}$-algebra $\mathcal{A}$ by taking

$$
\sigma_{t}(e(r))=e(r), \quad \sigma_{t}\left(\mu_{n}\right)=n^{i t} .
$$

Each element $\eta \in \hat{\mathbb{Z}}^{*} \simeq \operatorname{Hom}(\mathbb{Q} / \mathbb{Z}, \mathbb{Q} / \mathbb{Z})$ defines a representation $\pi_{\eta}$ of the $C^{*}$ algebra $\mathcal{A}$ as an algebra of operators on the Hilbert space $l^{2}\left(\mathbb{N}^{\times}\right)$via

$$
\pi_{\eta}(e(r)) \epsilon_{k}=\mathrm{e}^{2 \pi 1 \eta r} \varepsilon_{n}, \quad \pi_{\eta}\left(\mu_{n}\right) \varepsilon_{k}=\varepsilon_{n k}
$$

where $\left\{\varepsilon_{k}\right\}_{k \in \mathbb{N}^{\times}}$is the canonical basis for $l^{2}\left(\mathbb{N}^{\times}\right)$. For any of these representations the time evolution $\sigma_{t}$ can be implemented via the Hamiltonian $H\left(\varepsilon_{k}\right)=(\log k) \epsilon_{k}$ as:

$$
\pi_{\eta}\left(\sigma_{t}(a)\right)=\mathrm{e}^{1 t H} \pi_{\eta}(a) \mathrm{e}^{-1 t H} .
$$

The partition function of the system, defined as $\operatorname{Trace}\left(\mathrm{e}^{\beta H}\right)$ is then given by the Riemman zeta function $\zeta(\beta)$. The structure of equilibrium states for this system was studied in [2], where it is shown that for $0<\beta \leq 1$ there exist a unique $K M S_{\beta}$ state while for any $1<\beta \leq \infty$ there are infinitely many states and the space of extremal states $\mathcal{E}_{\beta}$ is homeomorphic to $\hat{\mathbb{Z}}^{*}$. The group $\hat{\mathbb{Z}}^{*}$ acts as a group of symmetries of the system $\left(\mathcal{A}, \sigma_{t}\right)$ and the induced action on $\mathcal{E}_{\beta}$ for $1<\beta \leq \infty$ is free and transitive. The arithmetic of abelian extensions of $\mathbb{Q}$ is encoded in this system in the following way:

Theorem 5.4 (Bost, Connes). For every $\varphi \in \mathcal{E}_{\infty}$ and every $a \in \mathcal{A}_{\mathbb{Q}}$ the value $\varphi(a)$ is algebraic over $\mathbb{Q}$. Moreover $\mathbb{Q}^{a b}$ is generated by values of this form and for all $\varphi \in \mathcal{E}_{\infty}, \gamma \in \operatorname{Gal}\left(\mathbb{Q}^{a b} \mid \mathbb{Q}\right)$ and $a \in \mathcal{A}_{\mathbb{Q}}$ one has

$$
\gamma \varphi(a)=\varphi\left(\Psi^{-1}(\gamma) a\right)
$$

where $\Psi: \mathcal{C}_{\mathbb{Q}} / D_{\mathbb{Q}}=\hat{\mathbb{Z}}^{*} \rightarrow \operatorname{Gal}\left(\mathbb{Q}^{a b} \mid \mathbb{Q}\right)$ is the class field theory isomorphism.

5.5 Algebraic and analytic endomotives. In this section we briefly survey the theory of endomotives as introduced in [5]. It provides a systematic way to construct associative algebras analogous to the rational sub-algebra of the BostConnes system in terms of arithmetic data. The resulting category provides an enlargement of the category of Artin motives over a number field to a category of arithmetic noncommutative spaces. 
Let $k$ be a number field. Given a projective system $\left\{X_{i}\right\}_{i \in I}$ of varieties in $\mathcal{V}^{0}(k), I$ a countable partially ordered set, we can consider the direct limit of algebras

$$
A=\underline{\lim }_{i} A_{i}
$$

where $X_{i}=\operatorname{Spec} A_{i}$. Assume that $S$ is an abelian semigroup acting by algebra endomorphisms on $A$ such that for any $s \in S$ the corresponding endomorphism $\rho_{s}$ induces an isomorphism

$$
A \simeq p_{s} A
$$

where $p_{s}=p_{s}^{2}=\rho_{s}(1)$.

Definition 5.5. An algebraic endomotive over $k$ is given by an associative algebra of the form

$$
\mathcal{A}_{k}=A \rtimes S
$$

corresponding a projective system $\left\{X_{i}\right\}_{i \in I}$ of varieties in $\mathcal{V}^{0}(k)$ and a semigroup $S$ as above.

Fix an algebraic closure $\bar{k}$ of $k$. For an algebraic endomotive $\mathcal{A}_{k}$ with corresponding projective system $\left\{X_{i}\right\}_{i \in I}$ the set of algebraic points of the pro-variety $X=\underline{\lim }_{i} X_{i}$ is given by the pro-finite (compact Hausdorff) space:

$$
X(\bar{k})=H_{k-a l g}(A, \bar{k}) .
$$

Given an embedding $\bar{k} \hookrightarrow \mathbb{C}$ we can identify $A$ with a sub-algebra of $C(X(\bar{k}))$. Pure states in $C(X(\bar{k}))$ attain algebraic values when restricted to $A$. Moreover, the natural action of the absolute Galois group $\operatorname{Gal}(\bar{k} / k)$ on $X(\bar{k})$ induces an action of $\operatorname{Gal}(\bar{k} / k)$ on the $C^{*}$-algebra

$$
\mathcal{A}=C(X(\bar{k})) \rtimes S
$$

in which the algebraic endomotive $\mathcal{A}_{k}$ embeds as a rational sub-algebra. An analytic endomotive is by definition a $C^{*}$-algebra of the above form. In the case of an endomotive given by abelian extensions of $k$ the corresponding action factors through $\operatorname{Gal}\left(k^{a b} / k\right)$ and the following result holds:

Theorem 5.6 (Connes, Consani, Marcolli). Let $\mathcal{A}_{k}$ be an algebraic endomotive over $k$ with corresponding projective system of varieties $\left\{X_{i}\right\}_{i \in I}$ and semigroup $S$. Assume that for each $i \in I$ the algebra $A_{i}$ is a finite product of normal abelian field extensions of $k$. Then the algebras $A$ and $\mathcal{A}_{k}$ are globally invariant under the action of $\operatorname{Gal}(\bar{k} / k)$ on $\mathcal{A}=C(X(\bar{k})) \rtimes S$. Moreover, any state $\varphi$ on $\mathcal{A}$ induced by a pure state on $C(X(\bar{k}))$ satisfies:

$$
\gamma \varphi(a)=\varphi\left(\gamma^{-1} a\right)
$$

for all $a \in \mathcal{A}_{k}$ and all $\gamma \in \operatorname{Gal}\left(k^{a b} / k\right)$. 
Example 5.7. Let $\left(Y, y_{0}\right)$ be a pointed algebraic variety over $k$ and let $S$ be an abelian semigroup of finite morphisms from $Y$ to itself. Assume moreover that any morphism in $S$ fixes $y_{0}$ and is unramified over $y_{0}$. For any $s \in S$ let

$$
X_{s}=\left\{y \in Y \mid s(y)=y_{0}\right\} .
$$

Order $S$ by divisibility, then the system $\left\{X_{s}\right\}_{s \in S}$ defines an algebraic endomotive over $k$ with $S$ as semigroup of endomorphisms.

Example 5.8. Let $\mathbb{G}_{m}$ denote the multiplicative group viewed as a variety over $\mathbb{Q}$. The power maps $z \mapsto z^{n}$ for $n \in \mathbb{N}^{\times}$define finite self morphisms on $\mathbb{G}_{m}$ fixing the point $1 \in \mathbb{G}_{m}$ and unramified over it. The algebraic endomotive associated to $\left(\mathbb{G}_{m}, 1\right)$ and $S=\mathbb{N}^{\times}$as in Example 5.7 is the arithmetic subalgebra $\mathcal{A}_{\mathbb{Q}}$ of the Bost-Connes system.

Example 5.9. Let $E$ be an elliptic curve with complex multiplication by an order $\mathcal{O}$ in an imaginary quadratic field $K$. Any endomorphism of $E$ in $\mathcal{O}^{\times}$fixes 0 and is unramified over it. The algebraic endomotive associated to $(E, 0)$ and $S=\mathcal{O}^{\times}$as in Example 5.7 is the arithmetic subalgebra of the quantum statistical mechanical system considered in [7]. This system encodes the class field theory of the field $K$.

An algebraic endomotive over $k$ defines a groupoid in a natural way. By considering groupoid actions satisfying a suitable étale condition it is possible to extend morphisms in $\operatorname{Corr}^{0}(k, F)$ in order to define correspondences between algebraic endomotives. The pseudo-abelian envelope of the category so obtained is the category of algebraic andomotives $\operatorname{EndMot}^{0}(k, F)$. By construction $\operatorname{Mot}^{0}(k, F)$ embeds as a full subcategory of $\operatorname{EndMot}^{0}(k, F)$.

The groupoid picture likewise allows to define a category of analytic endomotives $\mathbf{C}^{*} \mathbf{E n d M o t}^{0}(k, F)$ as the pseudo-abelian envelope of the category of correspondences between analytic endomotives. The map that assigns to an algebraic endomotive over $k$ with projective system $\left\{X_{i}\right\}_{i \in I}$ and semigroup $S$ the analytic endomotive $C(X(\bar{k})) \rtimes S, X=\underbrace{}_{\lim } X_{i}$, extends to a tensor functor:

$$
\operatorname{EndMot}^{0}(k, F) \rightarrow \mathbf{C}^{*} \operatorname{EndMot}^{0}(k, F)
$$

on which the Galois group $\operatorname{Gal}(\bar{k} / k)$ acts by natural transformations.

\section{References}

[1] E. Artin, J. Tate, Class field theory. W. A. Benjamin, Inc., New York-Amsterdam 1968.

[2] J.-B. Bost, A. Connes, Hecke algebras, type III factors and phase transitions with spontaneous symmetry breaking in number theory. Selecta Math. (N.S.) 1 (1995), no. $3,411-457$.

[3] O. Bratteli, D.W. Robinson, Operator algebras and quantum statistical mechanics. Springer, New York (1981). 
[4] A. Connes, Noncommutative geometry. Academic Press, 1994.

[5] A. Connes, C. Consani, M. Marcolli, Noncommutative geometry and motives: the thermodynamics of endomotives. Adv. Math. 214 (2007), no. 2, 761-831.

[6] A. Connes, M. Marcolli, Noncommutative geometry, quantum fields and motives. American Mathematical Society Colloquium Publications, 55. American Mathematical Society, Providence, RI; Hindustan Book Agency, New Delhi, 2008.

[7] A. Connes, M. Marcolli, N. Ramachandran, KMS states and complex multiplication. Selecta Math. (N.S.) 11 (2005) 325-347.

[8] A. Connes, M. Marcolli, N. Ramachandran, KMS states and complex multiplication. Part II, to appear in the Abel Symposium Volume.

[9] C. Consani, Noncommutative geometry and motives (a quoi servent les endomotifs?) arXiv:0711.0477v1 [math.QA] (2007).

[10] P. Deligne Valeurs de fonctions $L$ et périodes d'intégrales. With an appendix by N. Koblitz and A. Ogus. Proc. Sympos. Pure Math., XXXIII, Automorphic forms, representations and $L$-functions (Proc. Sympos. Pure Math., Oregon State Univ., Corvallis, Ore., 1977), Part 2, pp. 313-346, Amer. Math. Soc., Providence, R.I., 1979.

[11] M. Demazure, Motifs des variétés algébriques. Lecture Notes in Math. 180 (1971), 19-38., Séminaire Bourbaki 1969/70, exp. no. 365.

[12] W. Fulton, Intersection theory. Ergebnisse der Mathematik und ihrer Grenzgebiete (3), 2. Springer-Verlag, Berlin, 1984.

[13] R. Haag, N.M. Hugenholtz, M. Winnink, On the equilibrium states in quantum statistical mechanics. Comm. Math. Phys. 5 (1967) 215-236.

[14] R. Hartshorne, On the De Rham cohomology of algebraic varieties. Inst. Hautes tudes Sci. Publ. Math. No. 45 (1975), 5-99.

[15] J. Illusie, Crystalline cohomology. Motives (Seattle, WA, 1991), 43-70, Proc. Sympos. Pure Math., 55, Part 1, Amer. Math. Soc., Providence, RI, 1994.

[16] U. Jannsen, Equivalence relations on algebraic cycles. The arithmetic and geometry of algebraic cycles (Banff, AB, 1998), 225-260, NATO Sci. Ser. C Math. Phys. Sci., 548, Kluwer Acad. Publ., Dordrecht, 2000.

[17] U. Jannsen, Motives, numerical equivalence, and semi-simplicity. Invent. Math. 107 (1992), no. 3, 447-452.

[18] U. Jannsen, S. Kleiman, J-P Serre (Eds.) Motives. Proceedings of the AMS-IMSSIAM Joint Summer Research Conference held at the University of Washington, Seattle, Washington, July 20-August 2, 1991. Proceedings of Symposia in Pure Mathematics, 55, Parts 1 and 2. American Mathematical Society, Providence, RI, 1994.

[19] S. Kleiman Motives. Algebraic geometry, Oslo 1970 (Proc. Fifth Nordic SummerSchool in Math., Oslo, 1970), pp. 53-82. Wolters-Noordhoff, Groningen, (1972).

[20] S. Kleiman The standard conjectures. Motives (Seattle, WA, 1991), 3-20, Proc. Sympos. Pure Math., 55, Part 1, Amer. Math. Soc., Providence, RI, 1994.

[21] Y. Manin Correspondences, motifs and monoidal transformations. Mat. Sb. (N.S.) 77 (119) (1968) 475-507. 
[22] M. Marcolli, Arithmetic noncommutative geometry. With a foreword by Yuri Manin. University Lecture Series, 36. American Mathematical Society, Providence, RI, (2005).

[23] P. Samuel Relations d'équivalence en géométrie algébrique. Proc. Internat. Congress Math. 1958, 470-487. Cambridge Univ. Press, New York (1960).

[24] R. Sujatha Motives from a categorical point of view. Expository survey talk given at the Franco-Asian Conference on Motives held at IHES in 2006. (http://www.math.tifr.res.in/ sujatha/ihes.pdf) 


\title{
Motivic ideas in Noncommutative Geometry
}

\author{
An appendix by Matilde Marcolli
}

There has been in recent years a very fruitful interplay between ideas originally developed in the context of Grothendieck's theory of motives of algebraic varieties and techniques and notions arising in the context of noncommutative geometry.

Two main directions have become prominent: one based on treating noncommutative spaces as algebras, and importing motivic ideas by extending the notion of morphisms of noncommutative spaces to include Morita equivalences through correspondences realized by bimodules and other types of morphisms in larger categories like cyclic modules. This allows for cohomological methods based on cyclic (co)homology to be employed in a setting that provides an analog of the motivic ideas underlying the Weil proof of the Riemann hypothesis for function fields. It is this approach, developed in [8], [9], [12], that we focus on mostly in this survey. It concentrates on a category of noncommutative motives that are built out of the simplest class of motives of algebraic varieties, the Artin motives, which are motives of zero-dimensional algebraic varieties.

At the same time, there is a more general and very broad approach to motives in the noncommutative geometry setting, developed by Kaledin, Kontsevich, Tabuada, and others [20], [21], [33], based on the idea of representing noncommutative spaces as categories instead of algebras, and the related circle of ideas of derived algebraic geometry, [22], see also the short survey [25]. As we argue briefly in $\S 4$ below, one can expect that a merging of these two approaches will lead to some very interesting generalizations of some of the results that we review here.

\section{Noncommutative motives and cyclic cohomology}

In noncommutative geometry one encounters a problem that is very familiar in the context of algebraic geometry. Namely, if one thinks of noncommutative spaces as being described by associative algebras, then the category of algebras over a field with algebra homomorphisms is not abelian or even additive. Moreover, it is well known that morphisms of algebras are too restrictive a notion of morphisms for noncommutative spaces, as they do not account for the well known phenomenon of Morita equivalence.

Thus, one needs to embed the category of associative algebras with algebra homomorphisms inside an abelian (or at least pseudo-abelian) category with a larger collection of morphisms that include the correspondences given by tensoring with bimodules, as in the case of Morita equivalences. The objects of a category with these properties can be regarded as "noncommutative motives", in the same 
sense as the motives of algebraic varieties are the objects of an abelian category (or pseudo-abelian, or triangulated in the mixed case) that contains the category of algebraic varieties.

A first construction of such a category of noncommutative motives was obtained in [6], using the construction of cyclic modules.

One first defines the cyclic category $\Lambda$ as the category that has an object $[n]$ for each positive integer and has morphisms generated by the morphisms $\delta_{i}:[n-1] \rightarrow$ $[n], \sigma_{j}:[n+1] \rightarrow[n]$, and $\tau_{n}:[n] \rightarrow[n]$, with the relations

$$
\begin{gathered}
\delta_{j} \delta_{i}=\delta_{i} \delta_{j-1} \quad \text { for } i<j, \quad \sigma_{j} \sigma_{i}=\sigma_{i} \sigma_{j+1}, \quad i \leq j \\
\sigma_{j} \delta_{i}= \begin{cases}\delta_{i} \sigma_{j-1} & i<j \\
1_{n} & \text { if } i=j \text { or } i=j+1 \\
\delta_{i-1} \sigma_{j} & i>j+1 .\end{cases} \\
\tau_{n} \delta_{i}=\delta_{i-1} \tau_{n-1} \quad 1 \leq i \leq n, \quad \tau_{n} \delta_{0}=\delta_{n} \\
\tau_{n} \sigma_{i}=\sigma_{i-1} \tau_{n+1} \quad 1 \leq i \leq n, \quad \tau_{n} \sigma_{0}=\sigma_{n} \tau_{n+1}^{2} \\
\tau_{n}^{n+1}=1_{n} .
\end{gathered}
$$

Given a category $\mathcal{C}$, one defines as cyclic objects the covariant functors $\Lambda \rightarrow \mathcal{C}$. In particular, we consider the case where $\mathcal{C}=V e c t_{\mathbb{K}}$ is the category of vector spaces over a field $\mathbb{K}$ and we refer to the cyclic objects as cyclic modules, or $\mathbb{K}(\Lambda)$-modules.

In particular, consider a unital associative algebra $\mathcal{A}$ over a field $\mathbb{K}$. One associates to $\mathcal{A}$ a $\mathbb{K}(\Lambda)$-module $\mathcal{A}^{\natural}$, which is the covariant functor $\Lambda \rightarrow V e c t_{\mathbb{K}}$ that assigns to objects in $\Lambda$ the vector spaces

$$
[n] \Rightarrow \mathcal{A}^{\otimes(n+1)}=\mathcal{A} \otimes \mathcal{A} \cdots \otimes \mathcal{A}
$$

and to the generators of the morphisms of $\Lambda$ the linear maps

$$
\begin{gathered}
\delta_{i} \Rightarrow\left(a^{0} \otimes \cdots \otimes a^{n}\right) \mapsto\left(a^{0} \otimes \cdots \otimes a^{i} a^{i+1} \otimes \cdots \otimes a^{n}\right) \\
\sigma_{j} \Rightarrow\left(a^{0} \otimes \cdots \otimes a^{n}\right) \mapsto\left(a^{0} \otimes \cdots \otimes a^{i} \otimes 1 \otimes a^{i+1} \otimes \cdots \otimes a^{n}\right) \\
\tau_{n} \Rightarrow\left(a^{0} \otimes \cdots \otimes a^{n}\right) \mapsto\left(a^{n} \otimes a^{0} \otimes \cdots \otimes a^{n-1}\right) .
\end{gathered}
$$

The category of cyclic modules is an abelian category, and the construction above shows that one can embed inside it a copy of the category of associative algebras, hence the cyclic modules can be regarded as noncommutative motives. Notice that there are many more objects in the category of cyclic modules than those that come from associative algebras. For example, being an abelian category, kernels and cokernels of morphisms of cyclic modules are still cyclic modules even when, for instance, one does not have cokernels in the category of algebras.

Moreover, one sees that there are many more morphisms between cyclic modules than between algebras and that the type of morphisms one would like to have 
between noncommutative spaces, such as Morita equivalences, are included among the morphisms of cyclic modules.

In particular, all the following types of morphisms exist in the category of cyclic modules.

- Morphism of algebras $\phi: \mathcal{A} \rightarrow \mathcal{B}$ induce morphisms of cyclic modules $\phi^{\natural}$ : $\mathcal{A}^{\natural} \rightarrow \mathcal{B}^{\natural}$.

- Traces $\tau: \mathcal{A} \rightarrow \mathbb{K}$ induce morphisms of cyclic modules $\tau^{\natural}: \mathcal{A}^{\natural} \rightarrow \mathbb{K}^{\natural}$ by setting $\tau^{\natural}\left(x^{0} \otimes \cdots \otimes x^{n}\right)=\tau\left(x^{0} \cdots x^{n}\right)$.

- $\mathcal{A}-\mathcal{B}$ bimodules $\mathcal{E}$ induce morphisms of cyclic modules, $\mathcal{E}^{\natural}=\tau^{\natural} \circ \rho^{\natural}$, by composing $\rho: \mathcal{A} \rightarrow \operatorname{End}_{\mathcal{B}}(\mathcal{E})$ and $\tau: \operatorname{End}_{\mathcal{B}}(\mathcal{E}) \rightarrow \mathcal{B}$.

Moreover, it was shown in [6] that in the abelian category of cyclic modules the Ext functors recover cyclic cohomology of algebras by

$$
H C^{n}(\mathcal{A})=\operatorname{Ext}^{n}\left(\mathcal{A}^{\natural}, \mathbb{K}^{\natural}\right) .
$$

\section{Artin motives and the category of endomotives}

The simplest category of motives of algebraic varieties is the category of Artin motives, which corresponds to zero-dimensional varieties over a field $\mathbb{K}$ (which we take here to be a number field), with correspondences that are given by formal linear combinations of subvarieties $Z \subset X \times Y$ in the product. Because everything is zero-dimensional, in this case one does not have to worry about the different equivalence relations on cycles. Artin motives over $\mathbb{K}$ not only form an abelian category, but in fact a Tannakian category with motivic Galois group given by the absolute Galois group $\operatorname{Gal}(\overline{\mathbb{K}} / \mathbb{K})$.

The category of endomotives was introduced in [8] as a category of noncommutative spaces that are built out of towers of Artin motives with actions given by endomorphisms.

At the algebraic level, one considers as objects crossed product algebras $\mathcal{A}_{\mathbb{K}}=$ $A \rtimes S$, where $A$ is a commutative algebra over $\mathbb{K}$, obtained as a direct limit $A=$ $\lim _{\alpha} A_{\alpha}$ of finite dimensional reduced algebras over $\mathbb{K}$, which correspond under $\overrightarrow{X_{\alpha}}=\operatorname{Spec}\left(A_{\alpha}\right)$ to zero-dimensional algebraic varieties $X_{\alpha}$, Artin motives over $\mathbb{K}$. The direct limit $A$ of algebras corresponds to a pro-variety $X=\lim _{\alpha} X_{\alpha}$. The datum $S$ is a unital abelian semigroup, which acts on $A$ by endomorphisms with

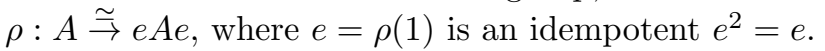

Morphisms between these objects are also constructed out of morphisms (correspondences) in the category of Artin motives, via projective limits, compatibly with the semigroup actions. More precisely, if $\mathcal{G}\left(X_{\alpha}, S\right)$ denotes the groupoid of the action of $S$ on $X$, the morphisms of endomotives are given by étale correspondences, which are formal linear combinations of $\mathcal{G}\left(X_{\alpha}, S\right)-\mathcal{G}\left(X_{\alpha^{\prime}}^{\prime}, S^{\prime}\right)$ spaces $Z$ for which the right action of $\mathcal{G}\left(X_{\alpha^{\prime}}^{\prime}, S^{\prime}\right)$ is étale. This means that, when representing 
$Z=\operatorname{Spec}(M)$, with $M$ an $\mathcal{A}_{\mathbb{K}}-\mathcal{A}_{\mathbb{K}}^{\prime}$ bimodule, $M$ is finite projective as a right $\mathcal{A}_{\mathbb{K}^{-}}$ module. Morphisms are then given by the $\mathbb{Q}$-linear space $M\left(\left(X_{\alpha}, S\right),\left(X_{\alpha^{\prime}}^{\prime}, S^{\prime}\right)\right)$ of formal linear combinations $U=\sum_{i} a_{i} Z_{i}$ of étale correspondences as above. The composition of morphisms is then given by the fibered product $Z \circ W=Z \times \times_{\mathcal{G}^{\prime}} W$ over the groupoid of the action of $S^{\prime}$ on $X^{\prime}$.

At the analytic level, one considers the topological space $\mathcal{X}=X(\overline{\mathbb{K}})$ with the action of the semigroup $S$. The topology is the one of the projective limit, which makes $X(\overline{\mathbb{K}})$ into a totally disconnected (Cantor-like) compact Hausdorff space. One can therefore consider the crossed product $C^{*}$-algebras $\mathcal{A}=C(X(\overline{\mathbb{K}})) \rtimes S=$ $C^{*}(\mathcal{G})$. One imposes a uniform condition, which provides a probability measure $\mu=\lim _{\longleftarrow} \mu_{\alpha}$ obtained using the counting measures on the $X_{\alpha}$, with $\frac{d \rho^{*} \mu}{d \mu}$ locally constant on $X(\overline{\mathbb{K}})$. Integration with respect to this measure gives a state $\varphi$ on the $C^{*}$-algebra $\mathcal{A}$.

One can also extend morphisms given by étale correspondences to this analytic setting. These are given by spaces $\mathcal{Z}$ with maps $g: \mathcal{Z} \rightarrow \mathcal{X}$ with discrete fiber and such that 1 is a compact operator on the right module $\mathcal{M}_{\mathcal{Z}}$ over $C(\mathcal{X})$ from the $C_{c}(\mathcal{G})$-valued inner product $\langle\xi, \eta\rangle(x, s):=\sum_{z \in g^{-1}(x)} \bar{\xi}(z) \eta(z \circ s)$.

For $\mathcal{G}-\mathcal{G}^{\prime}$ spaces defining morphisms of algebraic endomotives, one can consider $Z \mapsto Z(\overline{\mathbb{K}})=\mathcal{Z}$ and obtain a correspondence of analytic endomotives, with $C_{c}(\mathcal{Z})$ a right module over $C_{c}(\mathcal{G})$. These morphisms induce morphisms in the KK category and in the category of cyclic modules.

The analytic endomotive $\mathcal{A}$ is endowed with a Galois action of $G=\operatorname{Gal}(\overline{\mathbb{K}} / \mathbb{K})$, as an action on the characters $X(\overline{\mathbb{K}})=\operatorname{Hom}(A, \overline{\mathbb{K}})$ by

$$
A \stackrel{\chi}{\rightarrow} \overline{\mathbb{K}} \mapsto A \stackrel{\chi}{\rightarrow} \overline{\mathbb{K}} \stackrel{g}{\rightarrow} \overline{\mathbb{K}} .
$$

The action is also compatible with the endomorphisms action of $S$, since the latter acts on the characters by pre-composition. Thus, the Galois group acts by automorphisms of the crossed product algebra $\mathcal{A}=C(\mathcal{X}) \rtimes S$.

2.1 The Bost-Connes endomotive. A prototype example of an endomotive is the noncommutative space associated to the quantum statistical mechanical system constructed by Bost and Connes in [4]. A more transparent geometric interpretation of this noncommutative space as the moduli space of 1-dimensional $\mathbb{Q}$-lattices up to scale, modulo the equivalence relation of commensurability, was given in [11].

A $\mathbb{Q}$-lattice in $\mathbb{R}^{n}$ is a pair $(\Lambda, \phi)$ of a lattice $\Lambda \subset \mathbb{R}^{n}$ and a (possibly degenerate) labeling of its torsion points via a group homomorphism

$$
\phi: \mathbb{Q}^{n} / \mathbb{Z}^{n} \longrightarrow \mathbb{Q} \Lambda / \Lambda \text {. }
$$

In the special case where $\phi$ is an isomorphism one says that the $\mathbb{Q}$-lattice is invertible.

The equivalence relation of commensurability is defined by setting $\left(\Lambda_{1}, \phi_{1}\right) \sim$ $\left(\Lambda_{2}, \phi_{2}\right)$ whenever $\mathbb{Q} \Lambda_{1}=\mathbb{Q} \Lambda_{2}$ and $\phi_{1}=\phi_{2} \bmod \Lambda_{1}+\Lambda_{2}$. 
The quotient of the space of $\mathbb{Q}$-lattices by the commensurability relation is a "bad quotient" in the sense of ordinary geometry, but it can be described by a noncommutative space whose algebra of functions is the convolution algebra of the equivalence relation.

In the 1-dimensional case a $\mathbb{Q}$-lattice is specified by the data

$$
(\Lambda, \phi)=(\lambda \mathbb{Z}, \lambda \rho)
$$

for some $\lambda>0$ and some $\rho \in \operatorname{Hom}(\mathbb{Q} / \mathbb{Z}, \mathbb{Q} / \mathbb{Z})=\lim _{n} \mathbb{Z} / n \mathbb{Z}=\hat{\mathbb{Z}}$. If we consider the lattices up to scaling, we eliminate the factor $\overleftarrow{\lambda}$ and we are left with a space whose algebra of functions is $C(\hat{\mathbb{Z}})$.

The commensurability relation is then expressed by the action of the semigroup $\mathbb{N}=\mathbb{Z}_{>0}$ which maps $\alpha_{n}(f)(\rho)=f\left(n^{-1} \rho\right)$ when one can divide by $n$ and sets the result to zero otherwise.

The quotient of the space of 1-dimensional $\mathbb{Q}$-lattices up to scale by commensurability is then realized as a noncommutative space by the crossed product algebra $C(\hat{\mathbb{Z}}) \rtimes \mathbb{N}$. This can also be written as a convolution algebra for a partially defined action of $\mathbb{Q}_{+}^{*}$, with

$$
f_{1} * f_{2}(r, \rho)=\sum_{s \in \mathbb{Q}_{+}^{*}, s \rho \in \hat{\mathbb{Z}}} f_{1}\left(r s^{-1}, s \rho\right) f_{2}(s, \rho)
$$

with adjoint $f^{*}(r, \rho)=\overline{f\left(r^{-1}, r \rho\right)}$. This is the algebra of the groupoid of the commensurability relation. It is isomorphic to the Bost-Connes (BC) algebra of $[4]$.

As an algebra over $\mathbb{Q}$, it is given by $\mathcal{A}_{\mathbb{Q}, B C}=\mathbb{Q}[\mathbb{Q} / \mathbb{Z}] \rtimes \mathbb{N}$, and it has an explicit presentation by generators and relations of the form

$$
\begin{aligned}
& \mu_{n} \mu_{m}=\mu_{n m} \\
& \mu_{n} \mu_{m}^{*}=\mu_{m}^{*} \mu_{n} \quad \text { when }(n, m)=1 \\
& \mu_{n}^{*} \mu_{n}=1 \\
& \quad e(r+s)=e(r) e(s), \quad e(0)=1 \\
& \rho_{n}(e(r))=\mu_{n} e(r) \mu_{n}^{*}=\frac{1}{n} \sum_{n s=r} e(s)
\end{aligned}
$$

The $C^{*}$-algebra is then given by $C^{*}(\mathbb{Q} / \mathbb{Z}) \rtimes \mathbb{N}=C(\hat{\mathbb{Z}}) \rtimes \mathbb{N}$, where one uses the identification, via Pontrjagin duality, between $C(\hat{\mathbb{Z}})$ and $C^{*}(\mathbb{Q} / \mathbb{Z})$.

The time evolution of the $\mathrm{BC}$ quantum statistical mechanical system is given in terms of generators and relations by

$$
\sigma_{t}(e(r))=e(r), \quad \sigma_{t}\left(\mu_{n}\right)=n^{i t} \mu_{n}
$$

and it is generated by a Hamiltonian $H=\left.\frac{d}{d t} \sigma_{t}\right|_{t=0}$ with corresponding partition function $\operatorname{Tr}\left(e^{-\beta H}\right)=\zeta(\beta)$, in the representations on the Hilbert space $\ell^{2}(\mathbb{N})$ parameterized by the invertible $\mathbb{Q}$-lattices $\rho \in \hat{\mathbb{Z}}^{*}$. These representations $\pi_{\rho}$ on $\ell^{2}(N)$, 
for $\rho \in \hat{\mathbb{Z}}^{*}$, are given on generators by

$$
\mu_{n} \epsilon_{m}=\epsilon_{n m}, \quad \pi_{\rho}(e(r)) \epsilon_{m}=\zeta_{r}^{m} \epsilon_{m}
$$

where $\zeta_{r}=\rho(e(r))$ is a root of unity.

Given a $C^{*}$-algebra with time evolution, one can consider states, that is, linear functionals $\varphi: \mathcal{A} \rightarrow \mathbb{C}$ with $\varphi(1)=1$ and $\varphi\left(a^{*} a\right) \geq 0$, that are equilibrium states for the time evolution. As a function of a thermodynamic parameter (an inverse temperature $\beta$ ), these are specified by the KMS condition: $\varphi \in \mathrm{KMS}_{\beta}$ for some $0<\beta<\infty$ if $\forall a, b \in \mathcal{A}$ there exists a holomorphic function $F_{a, b}(z)$ on the strip $I_{\beta}=\{z \in \mathbb{C}: 0<\Im(z)<\beta\}$, continuous on the boundary $\partial I_{\beta}$, and such that, for all $t \in \mathbb{R}$,

$$
F_{a, b}(t)=\varphi\left(a \sigma_{t}(b)\right) \quad \text { and } \quad F_{a, b}(t+i \beta)=\varphi\left(\sigma_{t}(b) a\right) .
$$

In the case of the BC system the KMS states are classified in [4]: the low temperature extremal KMS states, for $\beta>1$ are of the form

$$
\varphi_{\beta, \rho}(a)=\frac{\operatorname{Tr}\left(\pi_{\rho}(a) e^{-\beta H}\right)}{\operatorname{Tr}\left(e^{-\beta H}\right)}, \quad \rho \in \hat{\mathbb{Z}}^{*},
$$

while at higher temperatures there is a unique KMS state.

At zero temperature the evaluations $\varphi_{\infty, \rho}(e(r))=\zeta_{r}$, which come from the projection on the kernel of the Hamiltonian,

$$
\varphi_{\infty, \rho}(a)=\left\langle\epsilon_{1}, \pi_{\rho}(a) \epsilon_{1}\right\rangle,
$$

exhibit an intertwining of the Galois action on the values of states on the arithmetic subalgebra and symmetries of the quantum statistical mechanical system: for $a \in$ $\mathcal{A}_{\mathbb{Q}, B C}$ and $\gamma \in \hat{\mathbb{Z}}^{*}$, one has

$$
\varphi_{\infty, \rho}(\gamma a)=\theta_{\gamma}\left(\varphi_{\infty, \rho}(a)\right)
$$

where

$$
\theta: \hat{\mathbb{Z}}^{*} \stackrel{\simeq}{\rightrightarrows} \operatorname{Gal}\left(\mathbb{Q}^{a b} / \mathbb{Q}\right)
$$

is the class field theory isomorphism.

The BC algebra is an endomotive with $A=\lim _{n} A_{n}$, for $A_{n}=\mathbb{Q}[\mathbb{Z} / n \mathbb{Z}]$ and the abelian semigroup action of $S=\mathbb{N}$ on $A=\mathbb{Q}[\overrightarrow{\mathbb{Q}} / \mathbb{Z}]$.

A more general class of endomotives was constructed in [8] using self-maps of algebraic varieties. One constructs a system $(A, S)$ from a collection $S$ of self maps of algebraic varieties $s: Y \rightarrow Y$ and their iterations, with $s\left(y_{0}\right)=y_{0}$ unbranched and $s$ of finite degree, by setting $X_{s}=s^{-1}\left(y_{0}\right)$ and taking the projective limit $X=\lim _{\longleftarrow} X_{s}=\operatorname{Spec}(A)$ under the maps

$$
\xi_{s, s^{\prime}}: X_{s^{\prime}} \rightarrow X_{s}, \quad \xi_{s, s^{\prime}}(y)=r(y), \quad s^{\prime}=r s \in S .
$$

The BC endomotive is a special case in this class, with $Y=\mathbb{G}_{m}$ with self maps $u \mapsto u^{k}$

$$
s_{k}: P\left(t, t^{-1}\right) \mapsto P\left(t^{k}, t^{-k}\right), \quad k \in \mathbb{N}, \quad P \in \mathbb{Q}\left[t, t^{-1}\right]
$$




$$
\xi_{k, \ell}(u(\ell))=u(\ell)^{k / \ell}, \quad u(\ell)=t \quad \bmod t^{\ell}-1 .
$$

One then has $X_{k}=\operatorname{Spec}\left(\mathbb{Q}\left[t, t^{-1}\right] /\left(t^{k}-1\right)\right)=s_{k}^{-1}(1)$ and $X=\lim _{k} X_{k}$ with

$$
u(\ell) \mapsto e(1 / \ell) \in \mathbb{Q}[\mathbb{Q} / \mathbb{Z}]
$$

One can identify the algebras $C(X(\overline{\mathbb{Q}}))=C(\hat{\mathbb{Z}})$.

2.2 Time evolution and KMS states. An object $(X, S)$ in the category of endomotives, constructed as above, determines the following data:

- A $C^{*}$-algebra $\mathcal{A}=C(\mathcal{X}) \rtimes S$.

- An arithmetic subalgebra $\mathcal{A}_{\mathbb{K}}=A \rtimes S$ defined over $\mathbb{K}$.

- A state $\varphi: \mathcal{A} \rightarrow \mathbb{C}$ from the uniform measure on the projective limit.

- An action of the Galois group by automorphisms $G \subset \operatorname{Aut}(\mathcal{A})$.

As shown in [8], see also $\S 4$ of [12], these data suffice to apply the thermodynamic formalism of quantum statistical mechanics. In fact, Tomita-Takesaki theory shows that one obtains from the state $\varphi$ a time evolution, for which $\varphi$ is a $\mathrm{KMS}_{1}$ state.

One starts with the GNS representation $\mathcal{H}_{\varphi}$. The presence of a cyclic and separating vector $\xi$ for this representation, so that $\mathcal{M} \xi$ and $\mathcal{M}^{\prime} \xi$ are both dense in $\mathcal{H}_{\varphi}$, with $\mathcal{M}$ the von Neumann algebra generated by $\mathcal{A}$ in the representation, ensures that one has a densely defined operator

$$
\begin{gathered}
S_{\varphi}: \mathcal{M} \xi \rightarrow \mathcal{M} \xi \quad a \xi \mapsto S_{\varphi}(a \xi)=a^{*} \xi \\
S_{\varphi}^{*}: \mathcal{M}^{\prime} \xi \rightarrow \mathcal{M}^{\prime} \xi \quad a^{\prime} \xi \mapsto S_{\varphi}^{*}\left(a^{\prime} \xi\right)=a^{\prime *} \xi
\end{gathered}
$$

which is closable and has a polar decomposition $S_{\varphi}=J_{\varphi} \Delta_{\varphi}^{1 / 2}$ with $J_{\varphi}$ a conjugatelinear involution $J_{\varphi}=J_{\varphi}^{*}=J_{\varphi}^{-1}$ and $\Delta_{\varphi}=S_{\varphi}^{*} S_{\varphi}$ a self-adjoint positive operator with $J_{\varphi} \Delta_{\varphi} J_{\varphi}=S_{\varphi} S_{\varphi}^{*}=\Delta_{\varphi}^{-1}$.

Tomita-Takesaki theory then shows that $J_{\varphi} \mathcal{M} J_{\varphi}=\mathcal{M}^{\prime}$ and $\Delta_{\varphi}^{-i t} \mathcal{M} \Delta_{\varphi}^{i t}=\mathcal{M}$, so that one obtains a time evolution (the modular automorphism group)

$$
\sigma_{t}(a)=\Delta_{\varphi}^{-i t} a \Delta_{\varphi}^{i t} \quad a \in \mathcal{M}
$$

for which the state $\varphi$ is a $\mathrm{KMS}_{1}$ state.

2.3 The classical points of a noncommutative space. Noncommutative spaces typically do not have points in the usual sense of characters of the algebra, since noncommutative algebras tend to have very few two-sided ideals. A good way to replace characters as a notion of points on a noncommutative space is by using extremal states, which in the commutative case correspond to extremal measures supported on points. While considering all states need not lead to a good 
topology on this space of points, in the presence of a natural time evolution, one can look at only those states that are equilibrium states for the time evolution. The notion of KMS states provides equilibrium states at a fixed temperature, or inverse temperature $\beta$. The extremal $\mathrm{KMS}_{\beta}$ states thus give a good working notion of points on a noncommutative space, with the interesting phenomenon that the set of points becomes temperature dependent and subject to phase transitions at certain critical temperatures.

In particular one can consider, depending on the inverse temperature $\beta$, that subset $\Omega_{\beta}$ of the extremal KMS states that is of Gibbs form, namely that is obtained from type $I_{\infty}$ factor representations. In typical cases, these arise as low temperature KMS-states, below a certain critical temperature and are then stable when going to lower temperatures, so that one has injective maps $c_{\beta^{\prime}, \beta}: \Omega_{\beta} \rightarrow \Omega_{\beta^{\prime}}$ for $\beta^{\prime}>\beta$.

For a state $\epsilon \in \Omega_{\beta}$ one has an irreducible representation $\pi_{\epsilon}: \mathcal{A} \rightarrow \mathcal{B}(\mathcal{H}(\epsilon))$, where the Hilbert space of the GNS representation decomposes as $\mathcal{H}_{\epsilon}=\mathcal{H}(\epsilon) \otimes \mathcal{H}^{\prime}$ with $\mathcal{M}=\{T \otimes 1: T \in \mathcal{B}(\mathcal{H}(\epsilon))\}$. The time evolution in this representation is generated by a Hamiltonian $\sigma_{t}^{\varphi}\left(\pi_{\epsilon}(a)\right)=e^{i t H} \pi_{\epsilon}(a) e^{-i t H}$ with $\operatorname{Tr}\left(e^{-\beta H}\right)<\infty$, so that the state can be written in Gibbs form

$$
\epsilon(a)=\frac{\operatorname{Tr}\left(\pi_{\epsilon}(a) e^{-\beta H}\right)}{\operatorname{Tr}\left(e^{-\beta H}\right)}
$$

The Hamiltonian $H$ is not uniquely determined, but only up to constant shifts $H \leftrightarrow H+c$, so that one obtains a real line bundle $\tilde{\Omega}_{\beta}=\{(\varepsilon, H)\}$, with $\lambda(\varepsilon, H)=$ $(\varepsilon, H+\log \lambda)$ for $\lambda \in \mathbb{R}_{+}^{*}$. The fibration $\mathbb{R}_{+}^{*} \rightarrow \tilde{\Omega}_{\beta} \rightarrow \Omega_{\beta}$ has a section $\operatorname{Tr}\left(e^{-\beta H}\right)=$ 1 , so it can be trivialized as $\tilde{\Omega}_{\beta} \simeq \Omega_{\beta} \times \mathbb{R}_{+}^{*}$.

Besides equilibrium KMS states, an algebra with a time evolution also gives rise to a dual system $(\hat{\mathcal{A}}, \theta)$, which is the algebra obtained by taking the crossed product with the time evolution, endowed with a scaling action by the dual group.

Namely, one considers the algebra: $\hat{\mathcal{A}}=\mathcal{A} \rtimes_{\sigma} \mathbb{R}$ given by functions $x, y \in$ $\mathcal{S}\left(\mathbb{R}, \mathcal{A}_{\mathbb{C}}\right)$ with convolution product $(x \star y)(s)=\int_{\mathbb{R}} x(t) \sigma_{t}(y(s-t)) d t$. One equivalently writes elements of $\hat{\mathcal{A}}$ formally as $\int x(t) U_{t} d t$, where $U_{t}$ are the unitaries that implement the $\mathbb{R}$ action $\sigma_{t}$.

The scaling action $\theta$ of $\lambda \in \mathbb{R}_{+}^{*}$ on $\hat{\mathcal{A}}$ is given by

$$
\theta_{\lambda}\left(\int x(t) U_{t} d t\right)=\int \lambda^{i t} x(t) U_{t} d t
$$

A point $(\varepsilon, H) \in \tilde{\Omega}_{\beta}$ determines an irreducible representation of $\hat{\mathcal{A}}$ by setting

$$
\pi_{\varepsilon, H}\left(\int x(t) U_{t} d t\right)=\int \pi_{\varepsilon}(x(t)) e^{i t H} d t,
$$

compatibly with the scaling action: $\pi_{\varepsilon, H} \circ \theta_{\lambda}=\pi_{\lambda(\varepsilon, H)}$. 
When restricting to those elements $x \in \hat{\mathcal{A}}_{\beta} \subset \hat{\mathcal{A}}$ that have an analytic continuation to the strip $I_{\beta}$ with rapid decay along the boundary, one obtains trace class operators [8]

$$
\pi_{\varepsilon, H}\left(\int x(t) U_{t} d t\right) \in \mathcal{L}^{1}(\mathcal{H}(\varepsilon))
$$

2.4 Restriction as a morphism of noncommutative motives. It is shown in [8] that one can define a restriction map from a noncommutative space to its classical points, where the latter are defined, as above, in terms of the low temperature extremal KMS states.

This restriction map does not exist as a morphism of algebras, but it does exist as a morphism in an abelian category of noncommutative motives that contains the category of algebras, namely the category of cyclic modules described above.

In fact, one can use the representations $\pi(x)(\varepsilon, H)$ and the trace class property to obtain a map

$$
\begin{gathered}
\hat{\mathcal{A}}_{\beta} \stackrel{\pi}{\longrightarrow} C\left(\tilde{\Omega}_{\beta}, \mathcal{L}^{1}\right) \stackrel{\operatorname{Tr}}{\longrightarrow} C\left(\tilde{\Omega}_{\beta}\right) \\
\pi(x)(\varepsilon, H)=\pi_{\varepsilon, H}(x) \quad \forall(\varepsilon, H) \in \tilde{\Omega}_{\beta},
\end{gathered}
$$

under a technical hypothesis on the vanishing of obstructions, see [8] and $\S 4$ of [12]. Because this map involves taking a trace, it is not a morphism in the category of algebras. However, as we have discussed above, traces are morphisms in the category of cyclic modules, so one regards the above map as a map of the corresponding cyclic modules,

$$
\begin{aligned}
& \hat{\mathcal{A}}_{\beta}^{\natural} \stackrel{\pi}{\rightarrow} C\left(\tilde{\Omega}_{\beta}, \mathcal{L}^{1}\right)^{\natural} \\
& \hat{\mathcal{A}}_{\beta}^{\natural} \stackrel{(\operatorname{Tro} \pi)^{\natural}}{\longrightarrow} C\left(\tilde{\Omega}_{\beta}\right)^{\natural} .
\end{aligned}
$$

This is equivariant for the scaling action of $\mathbb{R}_{+}^{*}$.

Moreover, we know by [6] that the category of cyclic modules is an abelian category. This means that the cokernel of this restriction map exists as a cyclic module, even though it does not come from an algebra. In [8] we denoted this cokernel as $D(\mathcal{A}, \varphi)=\operatorname{Coker}(\delta)$. One can compute its cyclic homology $H C_{0}(D(\mathcal{A}, \varphi))$, which also has an induced scaling action of $\mathbb{R}_{+}^{*}$, as well as an induced representation of the Galois group $G$, coming from the Galois representation on the endomotive $\mathcal{A}$.

This gives a space (not a noncommutative space but a noncommutative motive) $D(\mathcal{A}, \varphi)$ whose cohomology $H C_{0}(D(\mathcal{A}, \varphi))$ is endowed with a scaling and a Galois action. These data provide an analog in the noncommutative setting of the Frobenius action on étale cohomology in the context of motives of algebraic varieties. 
2.5 The Bost-Connes endomotive and the adeles class space. In [8] and [9] the motivic setting described above was applied in particular to the case of the Bost-Connes endomotive $\mathcal{A}=C(\hat{\mathbb{Z}}) \rtimes \mathbb{N}$ with the state given by the measure $\varphi(f)=\int_{\hat{\mathbb{Z}}} f(1, \rho) d \mu(\rho)$ and the resulting time evolution recovering the original time evolution of the Bost-Connes quantum statistical mechanical system, $\sigma_{t}(f)(r, \rho)=$ $r^{i t} f(r, \rho)$. In this case then the space of classical points is given by

$$
\tilde{\Omega}_{\beta}=\hat{\mathbb{Z}}^{*} \times \mathbb{R}_{+}^{*}=C_{\mathbb{Q}}=\mathbb{A}_{\mathbb{Q}}^{*} / \mathbb{Q}^{*}
$$

for small temperatures $\beta>1$.

The dual system is the groupoid algebra of the commensurability relation on $\mathbb{Q}$-lattices (considered not up to scaling) $\hat{\mathcal{A}}=C^{*}(\tilde{\mathcal{G}})$, where one identifies

$$
h(r, \rho, \lambda)=\int f_{t}(r, \rho) \lambda^{i t} U_{t} d t
$$

and the groupoid is parameterized by coordinates

$$
\tilde{\mathcal{G}}=\left\{(r, \rho, \lambda) \in \mathbb{Q}_{+}^{*} \times \hat{\mathbb{Z}} \times \mathbb{R}_{+}^{*}: r \rho \in \hat{\mathbb{Z}}\right\} .
$$

The Bost-Connes algebra is $\mathcal{A}=C^{*}(\mathcal{G})$ with $\mathcal{G}=\tilde{\mathcal{G}} / \mathbb{R}_{+}^{*}$ the groupoid of the commensurability relation on 1 -dimensional $\mathbb{Q}$-lattices up to scaling.

The combination of the scaling and Galois actions gives an action of $\hat{\mathbb{Z}}^{*} \times$ $\mathbb{R}_{+}^{*}=C_{\mathbb{Q}}$, since in the BC case the Galois action of $\operatorname{Gal}(\overline{\mathbb{Q}} / \mathbb{Q})$ factors through the abelianization. Characters $\chi$ of $\hat{\mathbb{Z}}^{*}$ determine projectors $p_{\chi}=\int_{\hat{\mathbb{Z}}^{*}} g \chi(g) d g$ where $p_{\chi}$ is an idempotent in the category of endomotives and in $\operatorname{End}_{\Lambda} D(\mathcal{A}, \varphi)$. Thus, one can considered the cohomology

$$
H C_{0}\left(p_{\chi} D(\mathcal{A}, \varphi)\right)
$$

of the range of this projector acting on the cokernel $D(\mathcal{A}, \varphi)$ of the restriction map.

2.6 Scaling as Frobenius in characteristic zero. The observation that a scaling action appears to provide a natural replacement for the Frobenius in characteristic zero is certainly not new to the work of [8] described above. In fact, perhaps the first very strong evidence for the parallels between scaling and Frobenius came from the comparative analysis, given in $\S 11$ of [1] of the number theoretic, characteristic $p$ method of Harder-Narasimhan [19] and the differential geometric method of Atiyah-Bott [1]. Both methods of [19] and [1] yield a computation of Betti numbers. In the number-theoretic setting this is achieved by counting points in the strata of a stratification, while in the Morse-theoretic approach one retracts strata onto the critical set. Both methods work because, on one side, one has a perfect Morse stratification, which essentially depends upon the fact that the strata 
are built out of affine spaces, and on the other hand one can effectively compute numbers of points in each stratum, for much the same reason. The explicit expressions obtained in both cases can be compared directly by a simple substitution that replaces the cardinality $q$ by a real variable $t^{2}$ and the Frobenius eigenvalues $\omega_{i}$ by $-t^{-1}$. In following this parallel between the characteristic $p$ and the characteristic zero case, one observes then that the role played by Frobenius in the first setting is paralleled by a scaling action in the characteristic zero world.

More closely related to the specific setting of the $\mathrm{BC}$ endomotive, one knows from the result of [15] that there is an analog of the BC system for function fields, where one works exclusively in positive characteristic. This starts with the observation in [14] that the quantum statistical mechanical system of [11], generalizing the BC system to 2-dimensional $\mathbb{Q}$-lattices, can be equivalently formulated in terms of Tate modules of elliptic curves with marked points,

$$
T E=H^{1}(E, \hat{\mathbb{Z}}) \quad \text { with } \quad \xi_{1}, \xi_{2} \in T E,
$$

with the commensurability relation implemented by isogenies. One then has a natural analog in the function field case. In fact, for $\mathbb{K}=\mathbb{F}_{q}(C)$, the usual equivalence of categories between elliptic curves and 2-dimensional lattices has an analog in terms of Drinfeld modules. One can then construct a noncommutative space, which can be described in terms of Tate modules of Drinfeld modules with marked points and the isogeny relation, or in the rank one case, in terms of 1-dimensional $\mathbb{K}$-lattices modulo commensurability. One constructs a convolution algebra, over a characteristic $p$ field $\mathbb{C}_{\infty}$, which is the completion of the algebraic closure of the completion $\mathbb{K}_{\infty}$ at a point $\infty$ of $C$.

One can extend to positive characteristic some of the main notions of quantum statistical mechanics, by a suitable redefinition of the notion of time evolutions and of their analytic continuations, which enter in the definition of KMS states. Over complex numbers, for $\lambda \in \mathbb{R}_{+}^{*}$ and $s=x+i y$ one can exponentiate as $\lambda^{s}=\lambda^{x} e^{i y \log \lambda}$. In the function field context, there is a similar exponentiation, for positive elements (with respect to a sign function) in $\mathbb{K}_{\infty}^{*}$ and for $s=(x, y) \in S_{\infty}:=\mathbb{C}_{\infty}^{*} \times \mathbb{Z}_{p}$, with $\lambda^{s}=x^{\operatorname{deg}(\lambda)}\langle\lambda\rangle^{y}$, with $\operatorname{deg}(\lambda)=-d_{\infty} v_{\infty}(\lambda)$, where $d_{\infty}$ is the degree of the point $\infty \in C$ and $v_{\infty}$ the corresponding valuation, and $\lambda=\operatorname{sign}(\lambda) u_{\infty}^{v_{\infty}(\lambda)}\langle\lambda\rangle$ the decomposition analogous to the polar decomposition of complex numbers, involving a sign function and a uniformizer $\mathbb{K}_{\infty}=\mathbb{F}_{q^{d \infty}}\left(\left(u_{\infty}\right)\right)$. The second term in the exponentiation is then given by

$$
\langle\lambda\rangle^{y}=\sum_{j=0}^{\infty}\left(\begin{array}{c}
y \\
j
\end{array}\right)(\langle\lambda\rangle-1)^{j}
$$

with the $\mathbb{Z}_{p}$-binomial coefficients

$$
\left(\begin{array}{c}
y \\
j
\end{array}\right)=\frac{y(y-1) \cdots(y-k+1)}{k !} .
$$


Exponentiation is an entire function $s \mapsto \lambda^{s}$ from $S_{\infty}$ to $\mathbb{C}_{\infty}^{*}$, with $\lambda^{s+t}=\lambda^{s} \lambda^{t}$, so one usually thinks of $S_{\infty}$ as a function field analog of the complex line with its polar decomposition $\mathbb{C}=U(1) \times \mathbb{R}_{+}^{*}$. One can extend the above to exponentiate ideals, $I^{s}=x^{\operatorname{deg}(I)}\langle I\rangle^{y}$. This gives an associated characteristic $p$ valued zeta function, the Goss $L$-function of the function field,

$$
Z(s)=\sum_{I} I^{-s},
$$

which is convergent in a "half plane" of $\left\{s=(x, y) \in S_{\infty}:|x|_{\infty}>q\right\}$.

The analog of a time evolution in this characteristic $p$ setting is then a continuous homomorphism $\sigma: \mathbb{Z}_{p} \rightarrow \operatorname{Aut}(\mathcal{A})$, where we think of $\mathbb{Z}_{p}$ as the line $\left\{s=(1, y) \in S_{\infty}\right\}$. In the case of the convolution algebra of 1-dimensional $\mathbb{K}$ lattices up to commensurability and scaling, a time evolution of this type is given using the exponentiation of ideals as

$$
\sigma_{y}(f)\left(L, L^{\prime}\right)=\frac{\langle I\rangle^{y}}{\langle J\rangle^{y}} f\left(L, L^{\prime}\right),
$$

for pairs $L \sim L^{\prime}$ of commensurable $\mathbb{K}$-lattices and the corresponding ideals. This gives a quantum statistical mechanical system in positive characteristic whose partition function is the Goss $L$-function.

One also has a notion of $\mathrm{KMS}_{x}$ functionals, which lack the positivity property of their characteristic zero version, but they have the defining property that $\varphi(a b)=$ $\varphi\left(\sigma_{x}(b) a\right)$, where $\sigma_{x}$ is the analytic continuation of the time evolution to $s=(x, 0)$.

Moreover, as shown in [15], one can construct a dual system in this function field setting as well, where the product on the dual algebra is constructed in terms of the momenta of the non-archimedean measure. The algebra of the dual system maps again naturally to the convolution algebra of the commensurability relation on 1-dimensional $\mathbb{K}$-lattices not up to scaling, which in turn can be expressed in terms of the adeles class space $\mathbb{A}_{\mathbb{K}} / \mathbb{K}^{*}$ of the function field. The algebra of the dual system also has a scaling action, exactly as in the characteristic zero case:

$$
\theta_{\lambda}(X)=\int_{H} \ell(s) \lambda^{s} U_{s} d \mu(s),
$$

where $H=G \times \mathbb{Z}_{p}$ with $G \subset \mathbb{C}_{\infty}^{*}$ and

$$
\begin{gathered}
\left.\theta_{\lambda}\right|_{G}(X)=\theta_{m}(X)=\int \ell(s) x^{-d_{\infty} m} U_{s} d \mu(s) \\
\left.\theta_{\lambda}\right|_{\mathbb{Z}_{p}}(X)=\theta_{\langle\lambda\rangle} \int \ell(s)\langle\lambda\rangle^{y} U_{s} d \mu(s)
\end{gathered}
$$

This action recovers the Frobenius action $F r^{\mathbb{Z}}$ as the part $\left.\theta_{\lambda}\right|_{G}$ of the scaling action, as well as the action of the inertia group, which corresponds to the part $\left.\theta_{\lambda}\right|_{\mathbb{Z}_{p}}$ 
2.7 The adele class space and the Weil proof. The adele class space is the bad quotient $\mathbb{A}_{\mathbb{K}} / \mathbb{K}^{*}$ of the adeles of a global field by the action of $\mathbb{K}^{*}$. Unlike the case of the action on the ideles $\mathbb{A}_{\mathbb{K}}^{*}$, which gives rise to a nice classical quotient, when one takes the action on the adeles the quotient is no longer described by a nice classical space, due to the ergodic nature of the action. However, it can be treated as a noncommutative space. In fact, this is the space underlying Connes' approach to the Riemann hypothesis via noncommutative geometry. Our purpose here is to describe the role of motivic ideas in noncommutative geometry, so we focus on the approach of [8] recalled in the previous section and we illustrate how the adeles class space relates to the algebra of the dual system of the Bost-Connes endomotive, as mentioned above for the function field analog.

A Morita equivalence given by compressing $C(\hat{\mathbb{Z}}) \rtimes \mathbb{N}=\left(C_{0}\left(\mathbb{A}_{\mathbb{Q}, f}\right) \rtimes \mathbb{Q}_{+}^{*}\right)_{\pi}$ with the projection given by the characteristic function $\pi$ of $\hat{\mathbb{Z}}$ can be used to identify the $\mathrm{BC}$ endomotive with the noncommutative quotient $\mathbb{A}_{\mathbb{Q}, f} / \mathbb{Q}_{+}^{*}$. The dual system is then identified with the noncommutative quotient $\mathbb{A}_{\mathbb{Q}} / \mathbb{Q}^{*}$, where $\mathbb{A}_{\mathbb{Q}}=\mathbb{A}_{\mathbb{Q}, f} \times \mathbb{R}^{*}$. The adeles class space $X_{\mathbb{Q}}:=\mathbb{A}_{\mathbb{Q}} / \mathbb{Q}^{*}$ is obtained by adding the missing point $0 \in \mathbb{R}$.

The way in which the adeles class space entered in Connes' work [7] on the Riemann zeta function was through a sequence of Hilbert spaces

$$
\begin{aligned}
& 0 \rightarrow L_{\delta}^{2}\left(\mathbb{A}_{\mathbb{Q}} / \mathbb{Q}^{*}\right)_{0} \stackrel{\mathfrak{E}}{\rightarrow} L_{\delta}^{2}\left(C_{\mathbb{Q}}\right) \rightarrow \mathcal{H} \rightarrow 0 \\
& \mathfrak{E}(f)(g)=|g|^{1 / 2} \sum_{q \in \mathbb{Q}^{*}} f(q g), \quad \forall g \in C_{\mathbb{Q}},
\end{aligned}
$$

where the space $L_{\delta}^{2}\left(\mathbb{A}_{\mathbb{Q}} / \mathbb{Q}^{*}\right)_{0}$ is defined by

$$
0 \rightarrow L_{\delta}^{2}\left(\mathbb{A}_{\mathbb{Q}} / \mathbb{Q}^{*}\right)_{0} \rightarrow L_{\delta}^{2}\left(\mathbb{A}_{\mathbb{Q}} / \mathbb{Q}^{*}\right) \rightarrow \mathbb{C}^{2} \rightarrow 0
$$

imposing the conditions $f(0)=0$ and $\hat{f}(0)=0$. The sequence above is compatible with the $C_{\mathbb{Q}}$ actions, so the operators

$$
U(h)=\int_{C_{\mathbb{Q}}} h(g) U_{g} d^{*} g \quad h \in \mathcal{S}\left(C_{\mathbb{Q}}\right)
$$

for compactly supported $h$, act on $\mathcal{H}$. The Hilbert space $\mathcal{H}$ can be decomposed $\mathcal{H}=\oplus_{\chi} \mathcal{H}_{\chi}$ according to characters $\chi$ of $\hat{\mathbb{Z}}^{*}$, and the scaling action of $\mathbb{R}_{+}^{*}$ on $\mathcal{H}_{\chi}=\left\{\xi \in \mathcal{H}: U_{g} \xi=\chi(g) \xi\right\}$ is generated by an operator $D_{\chi}$ with

$$
\operatorname{Spec}\left(D_{\chi}\right)=\left\{s \in i \mathbb{R} \mid L_{\chi}\left(\frac{1}{2}+i s\right)=0\right\}
$$

where $L_{\chi}$ is the L-function with Grössencharakter $\chi$ and in particular, the Riemann zeta function for $\chi=1$. 
The approach of Connes gives a semi-local trace formula, over the adeles class space restricted to a subset of finitely many places,

$$
\operatorname{Tr}\left(R_{\Lambda} U(h)\right)=2 h(1) \log \Lambda+\sum_{v \in S} \int_{\mathbb{Q}_{v}^{*}}^{\prime} \frac{h\left(u^{-1}\right)}{|1-u|} d^{*} u+o(1)
$$

where $R_{\Lambda}$ is a cutoff regularization and $\int^{\prime}$ is the principal value.

The trace formula should be compared to Weil's explicit formula in its distributional form:

$$
\hat{h}(0)+\hat{h}(1)-\sum_{\rho} \hat{h}(\rho)=\sum_{v} \int_{\mathbb{Q}_{v}^{*}}^{\prime} \frac{h\left(u^{-1}\right)}{|1-u|} d^{*} u .
$$

The geometric idea behind the Connes semi-local trace formula [7] is that it comes from the contributions of the periodic orbits of the action of $C_{\mathbb{Q}}$ on the complement of the classical points inside the adeles class space, $X_{\mathbb{Q}} \backslash C_{\mathbb{Q}}$. These are counted according to a version of the Guillemin-Sternberg distributional trace formula, originally stated for a flow $F_{t}=\exp (t v)$ on a manifold, implemented by transformations

$$
\left(U_{t} f\right)(x)=f\left(F_{t}(x)\right) \quad f \in C^{\infty}(M) .
$$

Under a transversality hypothesis which gives $1-\left(F_{t}\right)_{*}$ invertible, for

$$
\left(F_{t}\right)_{*}: T_{x} / \mathbb{R} v_{x} \rightarrow T_{x} / \mathbb{R} v_{x}=N_{x},
$$

the distributional trace formula takes the form

$$
\operatorname{Tr}_{d i s t r}\left(\int h(t) U_{t} d t\right)=\sum_{\gamma} \int_{I_{\gamma}} \frac{h(u)}{\left|1-\left(F_{u}\right)_{*}\right|} d^{*} u
$$

where $\gamma$ ranges over periodic orbits and $I_{\gamma}$ is the isotropy group, and $d^{*} u$ a measure with $\operatorname{covol}\left(I_{\gamma}\right)=1$. The distributional trace for a Schwartz kernel $(T f)(x)=$ $\int k(x, y) f(y) d y$ is of the form $\operatorname{Tr}_{d i s t r}(T)=\int k(x, x) d x$. For $(T f)(x)=f(F(x))$, this gives $(T f)(x)=\int \delta(y-F(x)) f(y) d y$.

The work of [8] and [9] presents a different but closely related approach, where one reformulates the noncommutative geometry method of [7] in a cohomological form with a motivic flavor, as we explained in the previous sections.

The restriction morphism $\delta=(\operatorname{Tr} \circ \pi)^{\natural}$ from the dual system of the BC endomotive to its classical points, both seen as noncommutative motives in the category of cyclic modules, can be equivalently written as

$$
\delta(f)=\sum_{n \in \mathbb{N}} f(1, n \rho, n \lambda)=\sum_{q \in \mathbb{Q}^{*}} \tilde{f}(q(\rho, \lambda))=\mathfrak{E}(\tilde{f})
$$

where $\tilde{f}$ is an extension by zero outside of $\hat{\mathbb{Z}} \times \mathbb{R}^{+} \subset \mathbb{A}_{\mathbb{Q}}$. 
The Hilbert space $L_{\delta}^{2}\left(\mathbb{A}_{\mathbb{Q}} / \mathbb{Q}^{*}\right)_{0}$ is replaced here by the cyclic-module $\hat{\mathcal{A}}_{\beta, 0}^{\natural}$. This requires different analytic techniques based on nuclear spaces, as in [31].

This provides a cohomological interpretation for the map $\mathfrak{E}$ and for the spectral realization, which is now associated to the scaling action $\theta$ on the cohomology $H C_{0}(D(\mathcal{A}, \varphi))$, which replaces the role of the Hilbert space $\mathcal{H}$ of $(2.3)$.

One has an action of $C_{\mathbb{Q}}=\mathbb{A}_{\mathbb{Q}}^{*} / \mathbb{Q}^{*}$ on $\mathcal{H}^{1}:=H C_{0}(D(\mathcal{A}, \varphi))$ by

$$
\vartheta(f)=\int_{C_{\mathbb{Q}}} f(g) \vartheta_{g} d^{*} g
$$

for $f \in S\left(C_{\mathbb{Q}}\right)$, a strong Schwartz space. Weil's explicit formula then has a global trace formula interpretation as

$$
\operatorname{Tr}\left(\left.\vartheta(f)\right|_{\mathcal{H}^{1}}\right)=\hat{f}(0)+\hat{f}(1)-\Delta \bullet \Delta f(1)-\sum_{v} \int_{\left(\mathbb{K}_{v}^{*}, e_{K_{v}}\right)}^{\prime} \frac{f\left(u^{-1}\right)}{|1-u|} d^{*} u
$$

The term $\Delta \bullet \Delta=\log |a|=-\log |D|$, with $D$ the discriminant for a number field, can be thought of as a self intersection of the diagonal, with the discriminant playing a role analogous to the Euler characteristic $\chi(C)$ of the curve for a function field $\mathbb{F}_{q}(C)$.

Thus, summarizing briefly the main differences between the approach of [7] and that of [8], [9], we have the following situation. In the trace formula for $\operatorname{Tr}\left(R_{\Lambda} U(f)\right)$ of [7] only the zeros on the critical line are involved and the Riemann hypothesis problem is equivalent to the problem of extending the semi-local trace formula to a global trace formula. This can be thought of in physical terms as a problem of passing from finitely many degrees of freedom to infinitely many, or equivalently from a quantum mechanical system to quantum field theory. In the setting of [8] and [9], instead, one has a global trace formula for $\operatorname{Tr}\left(\left.\vartheta(f)\right|_{\mathcal{H}^{1}}\right)$ and all the zeros of the Riemann zeta function are involved, since one is no longer working in the Hilbert space setting that is biased in favor of the critical line. In this setting the Riemann hypothesis becomes equivalent to a positivity statement

$$
\operatorname{Tr}\left(\left.\vartheta\left(f \star f^{\sharp}\right)\right|_{\mathcal{H}^{1}}\right) \geq 0 \quad \forall f \in S\left(C_{\mathbb{Q}}\right),
$$

where

$$
\left(f_{1} \star f_{2}\right)(g)=\int f_{1}(k) f_{2}\left(k^{-1} g\right) d^{*} g
$$

with the multiplicative Haar measure $d^{*} g$ and the adjoint is given by

$$
f^{\sharp}(g)=|g|^{-1} \overline{f\left(g^{-1}\right)} .
$$

This second setting makes for a more direct comparison with the algebrogeometric and motivic setting of the Weil proof of the Riemann hypothesis for function fields, which is based on similar ingredients: the Weil explicit formula and a positivity statement for the trace of correspondences. 
In a nutshell, the structure of the Weil proof for function fields is the following. The Riemann hypothesis for function fields $\mathbb{K}=\mathbb{F}_{q}(C)$ is the statement that the eigenvalues $\lambda_{n}$ of Frobenius have $\left|\lambda_{j}\right|=q^{1 / 2}$ in the zeta function

$$
\zeta_{\mathbb{K}}(s)=\prod_{\Sigma_{\mathbb{K}}}\left(1-q^{-n_{v} s}\right)^{-1}=\frac{P\left(q^{-s}\right)}{\left(1-q^{-s}\right)\left(1-q^{1-s}\right)},
$$

with $P(T)=\prod\left(1-\lambda_{n} T\right)$ the characteristic polynomial of the Frobenius Fr* acting on étale cohomology $H_{\mathrm{et}}^{1}\left(\bar{C}, \mathbb{Q}_{\ell}\right)$. This statement is shown to be equivalent to a positivity statement $\operatorname{Tr}\left(Z \star Z^{\prime}\right)>0$ for the trace of correspondences $Z=\sum_{n} a_{n} \mathrm{Fr}^{n}$ obtained from the Frobenius. Correspondences here are divisors $Z \subset C \times C$. These have a degree, codegree, and trace

$$
\begin{gathered}
d(Z)=Z \bullet(P \times C) \quad d^{\prime}(Z)=Z \bullet(C \times P) \\
\operatorname{Tr}(Z)=d(Z)+d^{\prime}(Z)-Z \bullet \Delta
\end{gathered}
$$

with $\Delta$ the diagonal in $C \times C$. One first adjusts the degree of the correspondence by trivial correspondences $C \times P$ and $P \times C$, then one applies Riemann-Roch to the divisor on the curve $P \mapsto Z(P)$ of $\operatorname{deg}=g$ and shows that it is linearly equivalent to an effective divisor. Then using $d\left(Z \star Z^{\prime}\right)=d(Z) d^{\prime}(Z)=g d^{\prime}(Z)=d^{\prime}\left(Z \star Z^{\prime}\right)$, one gets

$$
\begin{aligned}
& \operatorname{Tr}\left(Z \star Z^{\prime}\right)=2 g d^{\prime}(Z)+(2 g-2) d^{\prime}(Z)-Y \bullet \Delta \\
& \geq(4 g-2) d^{\prime}(Z)-(4 g-4) d^{\prime}(Z)=2 d^{\prime}(Z) \geq 0
\end{aligned},
$$

where $Z \star Z^{\prime}=d^{\prime}(Z) \Delta+Y$.

In the noncommutative geometry setting of [8] and [9] the role of the Frobenius correspondences is played by the scaling action of elements $g \in C_{\mathbb{K}}$ by

$$
Z_{g}=\left\{\left(x, g^{-1} x\right)\right\} \subset \mathbb{A}_{\mathbb{K}} / \mathbb{K}^{*} \times \mathbb{A}_{\mathbb{K}} / \mathbb{K}^{*}
$$

and more generally $Z(f)=\int_{C_{\mathbb{K}}} f(g) Z_{g} d^{*} g$ with $f \in S\left(C_{\mathbb{K}}\right)$. These correspondences also have a degree and codegree

$$
d(Z(f))=\hat{f}(1)=\int f(u)|u| d^{*} u
$$

with $d\left(Z_{g}\right)=|g|$ and

$$
d^{\prime}(Z(f))=d\left(Z\left(\bar{f}^{\sharp}\right)\right)=\int f(u) d^{*} u=\hat{f}(0) .
$$

Adjusting degree $d(Z(f))=\hat{f}(1)$ is possible by adding elements $h \in \mathcal{V}$, where $\mathcal{V}$ is the range of the restriction map $\delta=\operatorname{Tr} \circ \rho$,

$$
h(u, \lambda)=\sum_{n \in \mathbb{Z}^{\times}} \eta(n \lambda)
$$


with $\lambda \in \mathbb{R}_{+}^{*}$ and $u \in \hat{\mathbb{Z}}^{*}$, where $C_{\mathbb{Q}}=\hat{\mathbb{Z}}^{*} \times \mathbb{R}_{+}^{*}$. Indeed, one can find an element $h \in \mathcal{V}$ with $\hat{h}(1) \neq 0$ since Fubini's theorem fails,

$$
\int_{\mathbb{R}} \sum_{n} \eta(n \lambda) d \lambda \neq \sum_{n} \int_{\mathbb{R}} \eta(n \lambda) d \lambda=0 .
$$

One does not have a good replacement in this setting for principal divisors and linear equivalence, although one expects that the role of Riemann-Roch should be played by an index theorem in noncommutative geometry.

\section{Endomotives and $\mathbb{F}_{1}$-geometry}

In trying to exploit the analogies between function fields and number fields to import some of the ideas and methods of the Weil proof to the number fields context, one of the main questions is whether one can construct a geometric object playing the role of the product $C \times_{\mathbb{F}_{q}} C$ over which the Weil argument with correspondences $Z=\sum a_{n} F r^{n}$ is developed. We have seen in the previous section a candidate space built using noncommutative geometry, through the correspondences $Z_{g}$ of (2.5) on the adele class space. A different approach within algebraic geometry, aims at developing a geometry "over the field with one element" that would make it possible to interpret $\operatorname{Spec}(\mathbb{Z})$ as an analog of the curve, with a suitable space $\operatorname{Spec}(\mathbb{Z}) \times_{\mathbb{F}_{1}} \operatorname{Spec}(\mathbb{Z})$ playing the role of $C \times_{\mathbb{F}_{q}} C$.

The whole idea about a "field with one element", though no such thing can obviously exist in the usual sense, arises from early considerations of Tits on the behavior of the counting of points over finite fields in various examples of finite geometries. For instance, for $q=p^{k}$,

$$
\begin{gathered}
\# \mathbb{P}^{n-1}\left(\mathbb{F}_{q}\right)=\frac{\#\left(\mathbb{A}^{n}\left(\mathbb{F}_{q}\right) \backslash\{0\}\right)}{\# \mathbb{G}_{m}\left(\mathbb{F}_{q}\right)}=\frac{q^{n}-1}{q-1}=[n]_{q} \\
\# \operatorname{Gr}(n, j)\left(\mathbb{F}_{q}\right)=\#\left\{\mathbb{P}^{j}\left(\mathbb{F}_{q}\right) \subset \mathbb{P}^{n}\left(\mathbb{F}_{q}\right)\right\} \\
=\frac{[n]_{q} !}{[j]_{q} ![n-j]_{q} !}=\left(\begin{array}{c}
n \\
j
\end{array}\right)_{q}
\end{gathered}
$$

where one sets

$$
[n]_{q} !=[n]_{q}[n-1]_{q} \cdots[1]_{q}, \quad[0]_{q} !=1 .
$$

In all this cases, the expression one obtains when setting $q=1$ still makes sense and it appears to suggest a geometric replacement for each object. For example one obtains

$$
\begin{gathered}
\mathbb{P}^{n-1}\left(\mathbb{F}_{1}\right):=\text { finite set of cardinality } n \\
\operatorname{Gr}(n, j)\left(\mathbb{F}_{1}\right):=\text { set of subsets of cardinality } j .
\end{gathered}
$$

These observations suggestes the existence of something like a notion of algebraic geometry over $\mathbb{F}_{1}$, even though one need not have a direct definition of $\mathbb{F}_{1}$ itself. 
Further observations along these lines by Kapranov-Smirnov enriched the picture with a notion of "field extensions" $\mathbb{F}_{1^{n}}$ of $\mathbb{F}_{1}$, which are described in terms of actions of the monoid $\{0\} \cup \mu_{n}$, with $\mu_{n}$ the group of $n$-th roots of unity.

In this sense, one can say that a vector space over $\mathbb{F}_{1^{n}}$ is a pointed set $(V, v)$ endowed with a free action of $\mu_{n}$ on $V \backslash\{v\}$ and linear maps are just permutations compatible with the action.

So, as observed by Soulé and Kapranov-Smirnov, although one does not define $\mathbb{F}_{1^{n}}$ and $\mathbb{F}_{1}$ directly, one can make sense of the change of coefficients from $\mathbb{F}_{1}$ to $\mathbb{Z}$ as

$$
\mathbb{F}_{1^{n}} \otimes_{\mathbb{F}_{1}} \mathbb{Z}:=\mathbb{Z}\left[t, t^{-1}\right] /\left(t^{n}-1\right) .
$$

Various different approaches to $\mathbb{F}_{1}$-geometry have been developed recently by many authors: Soulé, Haran, Deitmar, Dourov, Manin, Toën-Vaquie, ConnesConsani, Borger, López-Peña and Lorscheid. Several of these viewpoints can be regarded as ways of providing descent data for rings from $\mathbb{Z}$ to $\mathbb{F}_{1}$. We do not enter here into a comparative discussion of these different approaches: a good overview of the current status of the subject is given in [24]. We are interested here in some of those versions of $\mathbb{F}_{1}$-geometry that can be directly connected with the noncommutative geometry approach described in the previous sections. We focus on the following approaches:

- Descent data determined by cyclotomic points (Soulé [32])

- Descent data by $\Lambda$-ring structures (Borger [3])

- Analytic geometry over $\mathbb{F}_{1}$ (Manin [27])

Soulé introduced in [32] a notion of gadgets over $\mathbb{F}_{1}$. These are triples of data $\left(X, \mathcal{A}_{X}, e_{x, \sigma}\right)$, where $X: \mathcal{R} \rightarrow$ Sets covariant functor from a category $\mathcal{R}$ of finitely generated flat rings, which can be taken to be the subcategory of rings generated by the group rings $\mathbb{Z}[\mathbb{Z} / n \mathbb{Z}] ; \mathcal{A}_{X}$ is complex algebra, with evaluation maps $e_{x, \sigma}$ such that, for all $x \in X(R)$ and $\sigma: R \rightarrow \mathbb{C}$ one has an algebra homomorphism $e_{x, \sigma}: \mathcal{A}_{X} \rightarrow \mathbb{C}$ with

$$
e_{f(y), \sigma}=e_{y, \sigma \circ f}
$$

for any ring homomorphism $f: R^{\prime} \rightarrow R$.

For example, affine varieties $V_{\mathbb{Z}}$ over $\mathbb{Z}$ define gadgets $X=G\left(V_{\mathbb{Z}}\right)$, by setting $X(R)=\operatorname{Hom}(O(V), R)$ and $\mathcal{A}_{X}=O(V) \otimes \mathbb{C}$.

An affine variety over $\mathbb{F}_{1}$ is then a gadget with $X(R)$ finite, and a variety $X_{\mathbb{Z}}$ with a morphism of gadgets $X \rightarrow G\left(X_{\mathbb{Z}}\right)$, with the property that, for all morphisms $X \rightarrow G\left(V_{\mathbb{Z}}\right)$ there exists a unique algebraic morphism $X_{\mathbb{Z}} \rightarrow V_{\mathbb{Z}}$, which functorially corresponds to the morphism of gadgets.

The Soulé data can be thought of as a descent condition from $\mathbb{Z}$ to $\mathbb{F}_{1}$, by regarding them as selecting among varieties defined over $\mathbb{Z}$ those that are determined by the data of their cyclotomic points $X(R)$, for $R=\mathbb{Z}[\mathbb{Z} / n \mathbb{Z}]$. This selects 
varieties that are very combinatorial in nature. For example, smooth toric varieties are geometries over $\mathbb{F}_{1}$ in this and all the other currently available flavors of $\mathbb{F}_{1}$-geometry.

Borger's approach to $\mathbb{F}_{1}$-geometry in [3] is based on a different way of defining descent conditions from $\mathbb{Z}$ to $\mathbb{F}_{1}$, using lifts of Frobenius, encoded in the algebraic structure of $\Lambda$-rings. This was developed by Grothendieck in the context of characteristic classes and the Riemann-Roch theorem, where it relates to operations in $K$-theory, but it can be defined abstractly in the following way.

For a ring $R$, whose underlying abelian group is torsion free, a $\Lambda$-ring structure is an action of the multiplicative semigroup $\mathbb{N}$ of positive integers by endomorphisms lifting Frobenius, namely, such that

$$
s_{p}(x)-x^{p} \in p R, \quad \forall x \in R .
$$

Morphisms of $\Lambda$-rings are ring homomorphisms $f: R \rightarrow R^{\prime}$ compatible with the actions, $f \circ s_{k}=s_{k}^{\prime} \circ f$.

The Bost-Connes endomotive, which is at the basis of the noncommutative geometry approach to the Riemann hypothesis, relates directly to both of these notions of $\mathbb{F}_{1}$ geometry in a very natural way.

3.1 Endomotives and Soulé's $\mathbb{F}_{1}$-geometry. The relation between the BC endomotive and Soulé's $\mathbb{F}_{1}$ geometry was investigated in [10]. One first considers a model over $\mathbb{Z}$ of the $\mathrm{BC}$ algebra. This requires eliminating denominators from the relations of the algebra over $\mathbb{Q}$. It can be done by replacing the crossed product by ring endomorphisms by a more subtle "crossed product" by correspondences. More precisely, one considers the algebra $\mathcal{A}_{\mathbb{Z}, B C}$ generated by $\mathbb{Z}[\mathbb{Q} / \mathbb{Z}]$ and elements $\mu_{n}^{*}$, $\tilde{\mu}_{n}$ with relations

$$
\begin{aligned}
& \tilde{\mu}_{n} \tilde{\mu}_{m}=\tilde{\mu}_{n m} \\
& \mu_{n}^{*} \mu_{m}^{*}=\mu_{n m}^{*} \\
& \mu_{n}^{*} \tilde{\mu}_{n}=n \\
& \tilde{\mu}_{n} \mu_{m}^{*}=\mu_{m}^{*} \tilde{\mu}_{n} \quad(n, m)=1 . \\
\mu_{n}^{*} x= & \sigma_{n}(x) \mu_{n}^{*} \quad \text { and } \quad x \tilde{\mu}_{n}=\tilde{\mu}_{n} \sigma_{n}(x),
\end{aligned}
$$

where $\sigma_{n}(e(r))=e(n r)$ for $r \in \mathbb{Q} / \mathbb{Z}$.

Notice that here the ring homomorphisms $\rho_{n}(x)=\mu_{n} x \mu_{n}^{*}$ are replaced by $\tilde{\rho}_{n}(x)=\tilde{\mu}_{n} x \mu_{n}^{*}$, which are no longer ring homomorphisms, but correspondences. The resulting "crossed product" is indicated by the notation $\mathcal{A}_{\mathbb{Z}, B C}=\mathbb{Z}[\mathbb{Q} / \mathbb{Z}] \rtimes_{\tilde{\rho}} \mathbb{N}$.

One then observes that roots of unity

$$
\underline{\mu}^{(k)}(R)=\left\{x \in R \mid x^{k}=1\right\}=\operatorname{Hom}_{\mathbb{Z}}\left(A_{k}, R\right)
$$

with $A_{k}=\mathbb{Z}\left[t, t^{-1}\right] /\left(t^{k}-1\right)$, can be organized as a system of varieties over $\mathbb{F}_{1}$ in two different ways. As an inductive system they define the multiplicative group $\mathbb{G}_{m}$ as a variety over $\mathbb{F}_{1}$ by taking

$$
\underline{\mu}^{(n)}(R) \subset \underline{\mu}^{(m)}(R), \quad n \mid m, \quad A_{m} \rightarrow A_{n}
$$


and by taking the complex algebra to be $\mathcal{A}_{X}=C\left(S^{1}\right)$.

As a projective system, which corresponds to the $\mathrm{BC}$ endomotive, one uses the morphisms $\xi_{m, n}: X_{n} \rightarrow X_{m}$

$$
\xi_{m, n}: \underline{\mu}^{(n)}(R) \rightarrow \underline{\mu}^{(m)}(R), \quad n \mid m
$$

and obtains a pro-variety

$$
\underline{\mu}^{\infty}(R)=\operatorname{Hom}_{\mathbb{Z}}(\mathbb{Z}[\mathbb{Q} / \mathbb{Z}], R),
$$

which arises from the projective system of affine varieties over $\mathbb{F}_{1}$

$$
\xi_{m, n}: \mathbb{F}_{1^{n}} \otimes_{\mathbb{F}_{1}} \mathbb{Z} \rightarrow \mathbb{F}_{1^{m}} \otimes_{\mathbb{F}_{1}} \mathbb{Z}
$$

where the complex algebra is taken to be $\mathcal{A}_{X}=\mathbb{C}[\mathbb{Q} / \mathbb{Z}]$.

The affine varieties $\mu^{(n)}$ over $\mathbb{F}_{1}$ are defined by gadgets $G(\operatorname{Spec}(\mathbb{Q}[\mathbb{Z} / n \mathbb{Z}]))$, which form a projective system of gadgets. The endomorphisms $\sigma_{n}$ of varieties over $\mathbb{Z}$, are also endomorphisms of gadgets and of $\mathbb{F}_{1}$-varieties.

The extensions $\mathbb{F}_{1^{n}}$ of Kapranov-Smirnov correspond to the free actions of roots of unity

$$
\zeta \mapsto \zeta^{n}, n \in \mathbb{N} \quad \text { and } \zeta \mapsto \zeta^{\alpha} \leftrightarrow e(\alpha(r)), \alpha \in \hat{\mathbb{Z}}
$$

and these can be regarded as the Frobenius action on $\mathbb{F}_{1 \infty}$. It should be noted that, indeed, in reductions mod $p$ of the integral Bost-Connes endomotive these do correspond to the Frobenius, so that one can consider the BC endomotive as describing the tower of extensions $\mathbb{F}_{1^{n}}$ together with the Frobenius action.

As shown in [10], one can obtain characteristic $p$ versions of the BC endomotive by separating out the parts

$$
\mathbb{Q} / \mathbb{Z}=\mathbb{Q}_{p} / \mathbb{Z}_{p} \times(\mathbb{Q} / \mathbb{Z})^{(p)}
$$

with denominators that are powers of $p$ and denominators that are prime to $p$. One then has a crossed product algebra

$$
\mathbb{K}\left[\mathbb{Q}_{p} / \mathbb{Z}_{p}\right] \rtimes p^{\mathbb{Z}^{+}}
$$

with endomorphisms $\sigma_{n}$ for $n=p^{\ell}$ and $\ell \in \mathbb{Z}^{+}$.

The Frobenius $\varphi_{\mathbb{F}_{p}}(x)=x^{p}$ of the field $\mathbb{K}$ in characteristic $p$ satisfies

$$
\left(\sigma_{p^{\ell}} \otimes \varphi_{\mathbb{F}_{p}}^{\ell}\right)(f)=f^{p^{\ell}}
$$

for $f \in \mathbb{K}[\mathbb{Q} / \mathbb{Z}]$ so that one has

$$
\left(\sigma_{p^{\ell}} \otimes \varphi_{\mathbb{F}_{p}}^{\ell}\right)(e(r) \otimes x)=e\left(p^{\ell} r\right) \otimes x^{p^{\ell}}=(e(r) \otimes x)^{p^{\ell}} .
$$

This shows that the BC endomorphisms restrict to Frobenius on the mod p reductions of the system: $\sigma_{p^{e}}$ induces the Frobenius correspondence on the pro-variety $\mu^{\infty} \otimes_{\mathbb{Z}} \mathbb{K}$. 
3.2 Endomotives and Borger's $\mathbb{F}_{1}$-geometry via $\Lambda$-rings. This is also the key observation in relating the $\mathrm{BC}$ endomotive to Borger's point of view [3] on $\mathbb{F}_{1}$-geometry. One sees, in fact, that the Bost-Connes endomotive is a direct limit of $\Lambda$-rings

$$
R_{n}=\mathbb{Z}\left[t, t^{-1}\right] /\left(t^{n}-1\right) \quad s_{k}(P)\left(t, t^{-1}\right)=P\left(t^{k}, t^{-k}\right),
$$

where the $\Lambda$-ring structures are given by the endomorphism action of $\mathbb{N}$, and the maps of the direct system are given again by the (2.1), (2.2). The action of $\hat{\mathbb{Z}}$ that combines the action of symmetries $\hat{\mathbb{Z}}^{*}$ by automorphisms of the BC system and the endomorphisms that give the $\Lambda$-ring structure is given by

$$
\alpha \in \hat{\mathbb{Z}}: \quad(\zeta: x \mapsto \zeta x) \mapsto\left(\zeta: x \mapsto \zeta^{\alpha} x\right),
$$

which transforms a free action by roots of unity $\zeta$ on a set of elements $x$ into a new action by $\zeta^{\alpha}$, where we identify $\hat{\mathbb{Z}}=\operatorname{Hom}(\mathbb{Q} / \mathbb{Z}, \mathbb{Q} / \mathbb{Z})$. This agrees with the notion of Frobenius over $\mathbb{F}_{1 \infty}$ proposed by Haran.

In fact, one can see the relation to $\Lambda$-rings more precisely by introducing multivariable generalizations of the $\mathrm{BC}$ endomotive as in [28].

One considers as varieties the algebraic tori $\mathbb{T}^{n}=\left(\mathbb{G}_{m}\right)^{n}$ with endomorphisms $\alpha \in M_{n}(\mathbb{Z})^{+}$, with $M_{n}(\mathbb{Z})^{+}$the semigroup of integer matrices with positive determinant. One constructs, as in the case of the $\mathrm{BC}$ endomotive the preimages

$$
X_{\alpha}=\left\{t=\left(t_{1}, \ldots, t_{n}\right) \in \mathbb{T}^{n} \mid s_{\alpha}(t)=t_{0}\right\}
$$

organized into a projective system with maps

$$
\begin{gathered}
\xi_{\alpha, \beta}: X_{\beta} \rightarrow X_{\alpha}, \quad t \mapsto t^{\gamma}, \quad \alpha=\beta \gamma \in M_{n}(\mathbb{Z})^{+} \\
t \mapsto t^{\gamma}=\sigma_{\gamma}(t)=\left(t_{1}^{\gamma_{11}} t_{2}^{\gamma_{12}} \cdots t_{n}^{\gamma_{1 n}}, \ldots, t_{1}^{\gamma_{n 1}} t_{2}^{\gamma_{n 2}} \cdots t_{n}^{\gamma_{n n}}\right) .
\end{gathered}
$$

The projective limit $X=\lim _{\alpha} X_{\alpha}$ carries a semigroup action of $M_{n}(\mathbb{Z})^{+}$.

One can then consider the algebra $C(X(\overline{\mathbb{Q}})) \cong \mathbb{Q}[\mathbb{Q} / \mathbb{Z}]^{\otimes n}$ with generators $e\left(r_{1}\right) \otimes \cdots \otimes e\left(r_{n}\right)$ and the crossed product

$$
\mathcal{A}_{n}=\mathbb{Q}[\mathbb{Q} / \mathbb{Z}]^{\otimes n} \rtimes_{\rho} M_{n}(\mathbb{Z})^{+}
$$

generated by $e(\underline{r})$ and $\mu_{\alpha}, \mu_{\alpha}^{*}$ with

$$
\begin{gathered}
\rho_{\alpha}(e(\underline{r}))=\mu_{\alpha} e(\underline{r}) \mu_{\alpha}^{*}=\frac{1}{\operatorname{det} \alpha} \sum_{\alpha(\underline{s})=\underline{r}} e(\underline{s}) \\
\sigma_{\alpha}(e(\underline{r}))=\mu_{\alpha}^{*} e(\underline{r}) \mu_{\alpha}=e(\alpha(\underline{r}))
\end{gathered}
$$

This corresponds to the action of the family of endomorphisms

$$
\sigma_{\alpha}(e(\underline{r}))=\mu_{\alpha}^{*} e(\underline{r}) \mu_{\alpha} .
$$

These multivariable versions relate to the $\Lambda$-rings notion of $\mathbb{F}_{1}$-geometry through a theorem of Borger-de Smit, which shows that every torsion free finite rank $\Lambda$-ring embeds in a finite product of copies of $\mathbb{Z}[\mathbb{Q} / \mathbb{Z}]$, where the action of $\mathbb{N}$ is compatible with the diagonal action $S_{n, \text { diag }} \subset M_{n}(\mathbb{Z})^{+}$in the multivariable BC endomotives. Thus, the multivariable BC endomotives are universal for $\Lambda$-rings. 
3.3 Endomotives and Manin's analytic geometry over $\mathbb{F}_{1}$. These multivariable generalizations of the $\mathrm{BC}$ endomotive introduced in [28] are also closely related to Manin's approach to analytic geometry over $\mathbb{F}_{1}$ of [27], which is based on the Habiro ring as a ring of analytic functions of roots of unity. The Habiro ring [18] is defined as the projective limit

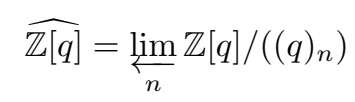

where

$$
(q)_{n}=(1-q)\left(1-q^{2}\right) \cdots\left(1-q^{n}\right)
$$

and one has morphisms $\mathbb{Z}[q] /\left((q)_{n}\right) \rightarrow \mathbb{Z}[q] /\left((q)_{k}\right)$ for $k \leq n$, since $(q)_{k} \mid(q)_{n}$. This ring has evaluation maps at roots of unity that are surjective ring homomorphisms

$$
e v_{\zeta}: \widehat{\mathbb{Z}[q]} \rightarrow \mathbb{Z}[\zeta]
$$

but which, combined, give an injective homomorphism

$$
e v: \widehat{\mathbb{Z}[q]} \rightarrow \prod_{\zeta \in \mathcal{Z}} \mathbb{Z}[\zeta]
$$

The elements of the Habiro ring also have Taylor series expansions at all roots of unity

$$
\tau_{\zeta}: \widehat{\mathbb{Z}[q]} \rightarrow \mathbb{Z}[\zeta][[q-\zeta]],
$$

which also are injective ring homomorphisms. Thus, they behave like "analytic functions on roots of unity".

As argued in [28], the Habiro ring provides then another model for the noncommutative geometry of the cyclotomic tower, replacing $\mathbb{Q}[\mathbb{Q} / \mathbb{Z}]$ with $\widehat{\mathbb{Z}[q]}$.

One considers endomorphisms $\sigma_{n}(f)(q)=f\left(q^{n}\right)$, which lift $P(\zeta) \mapsto P\left(\zeta^{n}\right)$ in $\mathbb{Z}[\zeta]$ through the evaluation maps $e v_{\zeta}$. This gives an action of $\mathbb{N}$ by endomorphisms and one can form a group crossed product

$$
\left.\mathcal{A}_{\mathbb{Z}, q}=\widehat{\mathbb{Z}[q]}\right]_{\infty} \rtimes \mathbb{Q}_{+}^{*}
$$

where $\mathcal{A}_{\mathbb{Z}, q}$ is generated by $\widehat{\mathbb{Z}[q]}$ and by elements $\mu_{n}$ and $\mu_{n}^{*}$ with

$$
\mu_{n} \sigma_{n}(f)=f \mu_{n}, \quad \mu_{n}^{*} f=\sigma_{n}(f) \mu_{n}^{*} .
$$

The ring $\widehat{\mathbb{Z}[q]}]_{\infty}=\cup_{N} A_{N}$, with $A_{N}$ generated by the $\mu_{N} f \mu_{N}^{*}$ satisfies

$$
\widehat{\mathbb{Z}[q]}]_{\infty}=\underset{n}{\lim }\left(\sigma_{n}: \widehat{\mathbb{Z}[q]} \rightarrow \widehat{\mathbb{Z}[q]}\right) \text {. }
$$

These maps are injective and determine automorphisms $\sigma_{n}: \widehat{\mathbb{Z}[q]_{\infty}} \rightarrow \widehat{\mathbb{Z}[q]_{\infty}}$. Another way to describe this is in terms of the $\operatorname{ring} \mathcal{P}_{\mathbb{Z}}$ of polynomials in $\mathbb{Q}$-powers $q^{r}$. One has

$$
\hat{\mathcal{P}}_{\mathbb{Z}}=\lim _{N} \mathcal{P}_{\mathbb{Z}} / \mathcal{J}_{N}
$$


where $\mathcal{J}_{N}$ is the ideal generated by $\left(q^{r}\right)_{N}=\left(1-q^{r}\right) \cdots\left(1-q^{r N}\right)$, with $r \in \mathbb{Q}_{+}^{*}$, and

$$
\widehat{\mathbb{Z}[q]}]_{\infty} \simeq \hat{\mathcal{P}}_{\mathbb{Z}}, \quad \mu_{n} f \mu_{n}^{*} \mapsto f\left(q^{1 / n}\right),
$$

where $\rho_{r}(f)(q)=f\left(q^{r}\right)$.

In [27], Manin also introduced multivariable versions of the Habiro ring,

$$
\mathbb{Z}\left[\widehat{q_{1}, \ldots,}, q_{n}\right]=\lim _{\overleftarrow{N}} \mathbb{Z}\left[q_{1}, \ldots, q_{n}\right] / I_{n, N},
$$

where $I_{n, N}$ is the ideal

$$
\left(\left(q_{1}-1\right)\left(q_{1}^{2}-1\right) \cdots\left(q_{1}^{N}-1\right), \ldots,\left(q_{n}-1\right)\left(q_{n}^{2}-1\right) \cdots\left(q_{n}^{N}-1\right)\right) .
$$

These again have evaluations at roots of unity

$$
e v_{\left(\zeta_{1}, \ldots, \zeta_{n}\right)}: \mathbb{Z}\left[\widehat{q_{1}, \ldots,} q_{n}\right] \rightarrow \mathbb{Z}\left[\zeta_{1}, \ldots, \zeta_{n}\right]
$$

and Taylor series expansions

$$
T_{Z}: \mathbb{Z}\left[\widehat{q_{1}, \ldots,} q_{n}\right] \rightarrow \mathbb{Z}\left[\zeta_{1}, \ldots, \zeta_{n}\right]\left[\left[q_{1}-\zeta_{1}, \ldots, q_{n}-\zeta_{n}\right]\right],
$$

for all $Z=\left(\zeta_{1}, \ldots, \zeta_{n}\right)$ in $\mathcal{Z}^{n}$, with $\mathcal{Z}$ the set of all roots of unity.

One can equivalently describe (3.1) as

$$
\mathbb{Z}\left[\widehat{q_{1}, \ldots,}, q_{n}\right]=\underset{\stackrel{\lim }{\leftarrow}}{\mathbb{Z}}\left[q_{1}, \ldots, q_{n}, q_{1}^{-1}, \ldots, q_{n}^{-1}\right] / \mathcal{J}_{n, N}
$$

where $\mathcal{J}_{n, N}$ is the ideal generated by the $\left(q_{i}-1\right) \cdots\left(q_{i}^{N}-1\right)$, for $i=1, \ldots, n$ and the $\left(q_{i}^{-1}-1\right) \cdots\left(q_{i}^{-N}-1\right)$. Conside then again the algebraic tori $\mathbb{T}^{n}=\left(\mathbb{G}_{m}\right)^{n}$, with algebra $\mathbb{Q}\left[t_{i}, t_{i}^{-1}\right]$. Using the notation

$$
t^{\alpha}=\left(t_{i}^{\alpha}\right)_{i=1, \ldots, n} \quad \text { with } \quad t_{i}^{\alpha}=\prod_{j} t_{j}^{\alpha_{i j}},
$$

we can define the semigroup action of $\alpha \in M_{n}(\mathbb{Z})^{+}$

$$
\begin{gathered}
q \mapsto \sigma_{\alpha}(q)=\sigma_{\alpha}\left(q_{1}, \ldots, q_{n}\right)= \\
\left(q_{1}^{\alpha_{11}} q_{2}^{\alpha_{12}} \cdots q_{n}^{\alpha_{1 n}}, \ldots, q_{1}^{\alpha_{n 1}} q_{2}^{\alpha_{n 2}} \cdots q_{n}^{\alpha_{n n}}\right)=q^{\alpha}
\end{gathered}
$$

analogous to the case of the multivariable $\mathrm{BC}$ endomotives discussed above, of which these constitute an analog in the setting of analytic $\mathbb{F}_{1}$-geometry. 


\section{DG-algebras and noncommutative motives}

The noncommutative motives we encountered so far in this overview are derived from two sources: the abelian category of cyclic modules and the category of endomotives, which are a very special kind of zero-dimensional noncommutative space combining Artin motives and endomorphism actions. More generally, one would like to incorporate higher dimensional algebraic varieties and correspondences given by algebraic cycles, together with their self maps, and construct larger categories of noncommutative spaces that generalize what we saw here in a zero-dimensional setting. In particular, this would be needed in order to generalize some of the results obtained so far for the Riemann zeta function using noncommutative geometry, like the trace formulae discussed above, to the more general context of $L$-functions of algebraic varieties and motives.

When one wishes to combine higher dimensional algebraic varieties with noncommutative spaces, one needs to pay attention to the substantially different way in which one treats the rings of functions in the two settings. This is not visible in a purely zero-dimensional case where one deals only with Artin motives. When one treats noncommutative spaces as algebras, one point of view is that one essentially only needs to deal with the affine case. The reason behind this is the fact that the way to describe in noncommutative geometry the gluing of affine charts, or any other kind of identification, is by considering the convolution algebra of the equivalence relation that implements the identifications. So, at the expense of no longer working with commutative algebras, one gains the possibility of always working with a single algebra of functions.

When one tries to combine noncommutative spaces with algebraic varieties, however, one wants to be able to deal directly with the algebro-geometric description of arbitrary quasi-projective varieties. This is where a more convenient approach is provided by switching the point of view from algebras to categories. The main result underlying the categorical approach to combining noncommutative geometry and motives is the fact that the derived category $\mathcal{D}(X)$ of quasicoherent sheaves on a quasiseparated quasicompact scheme $X$ is equivalent to the derived category $\mathcal{D}\left(\mathcal{A}^{\bullet}\right)$ of a DG-algebra $\mathcal{A}^{\bullet}$, which is unique up to derived Morita equivalence, see [2], [22]. Thus, passing to the setting of DG-algebras and DG-category provides a good setting where algebraic varieties can be treated, up to derived Morita equivalence, as noncommutative spaces.

A related question is the notion of correspondences between noncommutative spaces. We have seen in this short survey different notions of correspondences: morphisms of cyclic modules, among which one finds morphisms of algebras, bimodules, Morita equivalences, and traces. We also saw the correspondences associated to the scaling action of $C_{\mathbb{K}}$ on the noncommutative adeles class space $\mathbb{A}_{\mathbb{K}} / \mathbb{K}^{*}$. More generally, the problem of identifying the best class of morphisms of noncommutative spaces (or better of noncommutative motives) that accounts for all the desired features remains a question that is not settled in a completely satisfactory way. A comparison between different notions of correspondences in the analytic setting of 
KK-theory and in the context of derived algebraic geometry was given recently in [26], while a "motivic" category with correspondences based on noncommutative spaces defined as spectral triples and a version of smooth KK-theory was proposed in [30]. Again, a closer interplay between the analytic approach to noncommutative geometry via algebras, KK-theory, spectral triples, and such smooth differential notions, and the algebro-geometric approach via DG-categories and derived algebraic geometry is likely to play a crucial role in identifying the best notion of correspondences in noncommutative geometry.

\section{References}

[1] M.F. Atiyah, R. Bott, The Yang-Mills equations over Riemann surfaces. Philos. Trans. Roy. Soc. London Ser. A 308 (1983), no. 1505, 523-615.

[2] A. Bondal, M. Van den Bergh, Generators and representability of functors in commutative and noncommutative geometry, Mosc. Math. J. 3 (2003), 1-36.

[3] J. Borger, Lambda-rings and the field with one element, arXiv:0906.3146.

[4] J.B. Bost, A. Connes, Hecke algebras, type III factors and phase transitions with spontaneous symmetry breaking in number theory. Selecta Math. (N.S.) Vol.1 (1995) N.3, 411-457.

[5] D.C. Cisinski, G. Tabuada, Symmetric monoidal structure on non-commutative motives, arXiv:1001.0228.

[6] A. Connes, Cohomologie cyclique et foncteurs Ext ${ }^{n}$, C. R. Acad. Sci. Paris Sér. I Math. 296 (1983), no. 23, 953-958.

[7] A. Connes, Trace formula in noncommutative geometry and the zeros of the Riemann zeta function. Selecta Math. (N.S.) 5 (1999), no. 1, 29-106.

[8] A. Connes, C. Consani, M. Marcolli, Noncommutative geometry and motives: the thermodynamics of endomotives, Advances in Math. 214 (2) (2007), 761-831.

[9] A. Connes, C. Consani, M. Marcolli, The Weil proof and the geometry of the adeles class space, in "Algebra, Arithmetic, and Geometry: in honor of Yu.I.Manin" (Yu.Tschinkel, Yu.G.Zarhin, Eds.) Progress in Mathematics, Vol.270, Part I, Birkhäuser 2009, 339-406.

[10] A. Connes, C. Consani, M. Marcolli, Fun with $\mathbb{F}_{1}$, Journal of Number Theory, Vol.129 (2009), N.6, 1532-1561.

[11] A. Connes, M. Marcolli, From physics to number theory via noncommutative geometry, Part I: Quantum statistical mechanics of $\mathbb{Q}$-lattices, in Frontiers in Number Theory, Physics and Geometry, I pp.269-350, Springer Verlag, 2006.

[12] A. Connes, M. Marcolli, Noncommutative geometry, quantum fields and motives, Colloquium Publications, Vol.55, AMS, 2008.

[13] A. Connes, M. Marcolli, N. Ramachandran, KMS states and complex multiplication, Selecta Math. (N.S.) Vol.11 (2005) N.3-4, 325-347.

[14] A. Connes, M. Marcolli, N. Ramachandran, KMS states and complex multiplication. II. in "Operator Algebras: The Abel Symposium 2004", pp.15-59, Abel Symp., 1, Springer Verlag, 2006. 
[15] C. Consani, M. Marcolli, Quantum statistical mechanics over function fields, Journal of Number Theory 123 (2) (2007) 487-528.

[16] G. Cornelissen, M. Marcolli, Quantum statistical mechanics, L-series and anabelian geometry, in preparation.

[17] E. Ha, F. Paugam, Bost-Connes-Marcolli systems for Shimura varieties. I. Denitions and formal analytic properties, IMRP Int. Math. Res. Pap. (2005), no. 5, 237-286.

[18] K. Habiro, Cyclotomic completions of polynomial rings, Publ. RIMS Kyoto Univ. (2004) Vol.40, 1127-1146.

[19] G. Harder, M.S. Narasimhan, On the cohomology groups of moduli spaces of vector bundles on curves, Math. Ann. 212 (1974/75), 215-248.

[20] D. Kaledin, Motivic structures in non-commutative geometry, ICM-2010 talk, arXiv:1003.3210.

[21] D. Kaledin, Cartier isomorphism and Hodge theory in the non-commutative case, in "Arithmetic geometry", 537-562, Clay Math. Proc., 8, AMS, 2009.

[22] B. Keller, On differential graded categories, in "International Congress of Mathematicians". Vol. II, 151-190, Eur. Math. Soc., Zürich, 2006.

[23] M. Kontsevich, Notes on motives in finite characteristic, in "Algebra, Arithmetic, and Geometry: in honor of Yu.I.Manin" (Yu.Tschinkel, Yu.G.Zarhin, Eds.) Progress in Mathematics, Vol.270, Part II, Birkhäuser 2009, 213-248.

[24] J. López Peña, O. Lorscheid, Mapping $\mathbb{F}_{1}$-land: An overview of geometries over the field with one element, arXiv:0909.0069.

[25] S. Mahanta, Noncommutative geometry in the framework of differential graded categories, in "Arithmetic and geometry around quantization", Progress in Mathematics, Vol.279, Birkhäuser 2010, 253-276.

[26] S. Mahanta, Noncommutative correspondence categories, simplicial sets and pro $C^{*}$. algebras, arXiv:0906.5400.

[27] Yu.I. Manin, Cyclotomy and analytic geometry over $\mathbb{F}_{1}$, arXiv:0809.1564.

[28] M. Marcolli, Cyclotomy and endomotives, P-Adic Numbers, Ultrametric Analysis and Applications, Vol.1 (2009) N.3, 217-263.

[29] M. Marcolli, Arithmetic noncommutative geometry, University Lectures Series, Vol.35, AMS, 2005.

[30] B. Mesland, Unbounded biviariant $K$-theory and correspondences in noncommutative geometry, arXiv:0904.4383.

[31] R. Meyer, On a representation of the idele class group related to primes and zeros of L-functions, Duke Math. J. 127 (2005), no. 3, 519-595.

[32] C. Soulé, Les variétés sur le corps à un élément, Mosc. Math. J. Vol.4 (2004) N.1, 217-244.

[33] G. Tabuada, Universal suspension via non-commutative motives, arXiv:1003.4425. 
Sujatha Ramdorai, School of Mathematics, Tata Institute of Fundamental Research, Homi Bhabha Road, Colaba, Mumbai 400 005, India

E-mail: sujatha@math.tifr.res.in

Jorge Plazas, Department of Mathematics, University of Utrecht, P.O.Box 80010, NL-3508 TA Utrecht, The Netherlands

E-mail: j.a.plazasvargas@uu.nls

Matilde Marcolli, Mathematics Department, Mail Code 253-37, Caltech, 1200 E.California Blvd. Pasadena, CA 91125, USA

E-mail: matilde@caltech.edu 